https://helda.helsinki.fi

\title{
Strong anisotropy of superfluid He-4 counterflow turbulence
}

\author{
Biferale, L.
}

2019-10-28

Biferale , L , Khomenko , D , L'vov , V S , Pomyalov , A , Procaccia , I \& Sahoo , G 2019 , ' Strong anisotropy of superfluid He-4 counterflow turbulence ', Physical Review B , vol. 100 , no. 13 , 134515 . https://doi.org/10.1103/PhysRevB.100.134515

http://hdl.handle.net/10138/307183

https://doi.org/10.1103/PhysRevB.100.134515

cc_by

publishedVersion

Downloaded from Helda, University of Helsinki institutional repository.

This is an electronic reprint of the original article.

This reprint may differ from the original in pagination and typographic detail.

Please cite the original version. 


\title{
Strong anisotropy of superfluid ${ }^{4} \mathrm{He}$ counterflow turbulence
}

\author{
L. Biferale, ${ }^{1}$ D. Khomenko $\odot,{ }^{2}$ V. S. L’vov, ${ }^{3}$ A. Pomyalov $\odot,{ }^{3}$ I. Procaccia,,${ }^{3,4}$ and G. Sahoo $\odot^{5}$ \\ ${ }^{1}$ Department of Physics and INFN, University of Rome, Tor Vergata, I-Roma, Italy \\ ${ }^{2}$ Laboratoire de Physique, Département de Physique de l'ENS, École Normale Supérieure, PSL Research University, Sorbonne Universités, \\ CNRS, F-75005 Paris, France \\ ${ }^{3}$ Department of Chemical and Biological Physics, Weizmann Institute of Science, Rehovot, Israel \\ ${ }^{4}$ Center for OPTical IMagery Analysis and Learning, Northwestern Polytechnical University, Xi'an, 710072 China \\ ${ }^{5}$ Department of Mathematics and Statistics and Department of Physics, University of Helsinki, Finland
}

(Received 28 August 2019; published 28 October 2019)

\begin{abstract}
We report on a combined theoretical and numerical study of counterflow turbulence in superfluid ${ }^{4} \mathrm{He}$ in a wide range of parameters. The energy spectra of the velocity fluctuations of both the normal-fluid and superfluid components are strongly anisotropic. The angular dependence of the correlation between velocity fluctuations of the two components plays the key role. A selective energy dissipation intensifies as scales decrease, with the streamwise velocity fluctuations becoming dominant. Most of the flow energy is concentrated in a wave-vector plane which is orthogonal to the direction of the counterflow. The phenomenon becomes more prominent at higher temperatures as the coupling between the components depends on the temperature and the direction with respect to the counterflow velocity.
\end{abstract}

DOI: $10.1103 /$ PhysRevB.100.134515

\section{INTRODUCTION}

Below a critical temperature $T_{\lambda} \approx 2.17 \mathrm{~K}$ liquid ${ }^{4} \mathrm{He}$ behaves as a quantum fluid [1,2], consisting of an inviscid superfluid, associated with the quantum ground state, and a gas of thermal excitations which make up the viscous normal fluid. Quantum mechanics [4] constrains the rotational motion of the superfluid in ${ }^{4} \mathrm{He}$ to discrete Ångström-width quantum vortex lines of fixed circulation. The thermal excitations scatter on a dense tangle of these vortices, thus inducing a mutual friction force between the normal fluid and the superfluid.

Turbulent superfluid helium in a channel with a temperature gradient is a subject of extensive research for many decades [1-10]. In such a setting, so-called "counterflow," the normal fluid flows from the hot end of the channel to the cold end while the superfluid flows in the opposite direction. Most attention was devoted so far to the measurement and the analysis of the density of vortex lines and to the mutual friction between the components.

Recent advances in the visualization techniques offer for the first time a direct access to the statistics of the velocity fluctuations of the normal fluid [11-13] and the superfluid [14-16]. It was shown that the large-scale statistics of the normal fluid in the counterflow is very different [11-13] from the statistics of classical fluids. The theoretical analysis [17-19] highlighted the importance of correlations between the superfluid and the normal-fluid components, which lead to the energy spectra of both components being steeper than their classical counterparts. Moreover, we have recently shown [19] that the direction of the mean relative velocity plays an important role; the correlation between the ${ }^{4} \mathrm{He}$ components decays slower for eddies stretched along the counterflow velocity. In contrast, the correlation of eddies, which are elongated in the orthogonal direction, decay faster, leading to their enhanced energy loss. As a result of this directionally preferred energy dissipation, the velocity fluctuations consist mostly of the streamwise component, while most of the flow energy is concentrated in the wave-vector plane orthogonal to the counterflow.

Here we consider this phenomenon further and study its consequences in further detail. The paper is organized as follows: In Sec. II we provide a sketch of a theory of counterflow turbulence with a stress on its anisotropy. In Sec. II A we introduce the basic set of coarse-grained equations for the counterflow. These are used for the theoretical analysis and for the numerical simulations.

In Sec. II B we clarify how various approaches to the statistical description of the anisotropy energy surface are related. Next, in Sec. IIC, we discuss the physical origin of the strong spectral anisotropy in counterflow turbulence. In Sec. III we present the results of the direct numerical simulations (DNS) of the two-fluid coarse-grained Eq. (1a). The main conclusion is that the analytical predictions are confirmed. In Sec. III A we discuss the simulation parameters and the numerical procedure. Next, in Sec. III B, we use standard statistical characteristics: one-dimensional (1D) energy spectra and cross-correlation functions, averaged over a spherical surface of radius $k$ (i.e., over all directions of vector $\boldsymbol{k}$ ) to provide an overview of spectral properties of ${ }^{4} \mathrm{He}$ counterflow. We find that at the small- $k$ regime, the normalfluid and superfluid velocity components are indeed well correlated. As expected, mutual friction plays a secondary role and the spherically averaged spectra are similar to the spectra in the ${ }^{4} \mathrm{He}$-coflow turbulence [20], being only slightly steeper than the Kolmogorov-1941 (K41) spectra of classical hydrodynamic turbulence. On the other hand, at relatively large $k$ the fluid components are practically uncorrelated; mutual friction provides a leading contribution to the energy dissipation and the counterflow spectra are similar to those in 
${ }^{3} \mathrm{He}$ superfluid turbulence with the normal-fluid component at rest $[21,22]$. The spectra become strongly suppressed in comparison to K41 energy spectra.

The similarities between the inherently anisotropic counterflow energy spectra and the isotropic spectra in the turbulent ${ }^{4} \mathrm{He}$-coflow and ${ }^{3} \mathrm{He}$ are, however, superficial. To expose the differences, we discuss in Sec. III C the two-dimensional (2D) energy spectra which depend, besides the wave number $k$, upon the angle $\theta$ between the wave vector $\boldsymbol{k}$ and the counterflow velocity $\boldsymbol{U}_{\text {ns }}$. Here we find that the spectra become more and more anisotropic with increasing $k$, being confined in $\boldsymbol{k}$ space to a small range $\cos \theta<0.1$, i.e., near the wave-vector plane which is orthogonal to $\boldsymbol{U}_{\mathrm{ns}}$. This effect becomes stronger with increasing temperature. The tensor structure of the energy spectra, considered in Sec. III D, is also temperature dependent: The small-scale turbulent velocity fluctuations are dominated by only one vector component, parallel to $\boldsymbol{U}_{\mathrm{ns}}$, becoming more so at higher temperature. Further, we compare several variants of differently averaged 1D spectra (Sec. III E) and structure functions (Sec. III F) to expose other aspects of the spectral anisotropy in connection with possible experimental observations.

In Sec. IV we summarize our findings: Counterflows exhibit strongly anisotropic energy distributions. The energy spectra are localized near a direction that is orthogonal to the counterflow. The phenomenon is similar to atmospheric turbulence with a strong stable stratification or to rotational turbulence [23-28]. On the other hand, the tensor structures of these two types of quasi-2D turbulence are quite the opposite: In atmospheric turbulence the vertical component of the turbulent velocity fluctuations is suppressed by the stratification and only the two horizontal components are dominant [23]. In the counterflow turbulence the main contribution to the turbulent energy comes from one streamwise velocity projection, while the two cross-steam velocity projections are strongly suppressed. The observed phenomenon is mild at low temperatures and becomes more prominent as the temperature increases. We confirm, in agreement with Ref. [13], that the structure functions of the turbulent velocities in the counterflow do not reflect in a quantitative manner the underlying energy spectra. However, the relative magnitude of the structure functions, measured in different directions, may qualitatively reflect the presence of the spectral anisotropy.

\section{QUALITATIVE ANALYSIS OF ANISOTROPIC COUNTERFLOW TURBULENCE}

As we mentioned in the introduction, one of the important properties of superfluid ${ }^{4} \mathrm{He}$ is the quantization of vorticity, which concentrates on vortex lines of core radius $a_{0} \approx$ $10^{-8} \mathrm{~cm}$ with fixed circulation $\kappa=h / M \approx 10^{-3} \mathrm{~cm}^{2} / \mathrm{s}$. Here $h$ is Planck's constant and $M$ is the mass of the ${ }^{4} \mathrm{He}$ atom [4]. A complex tangle of these vortex lines with a typical intervortex distance [3] $\ell \sim 10^{-4}-10^{-2} \mathrm{~cm}$ is a manifestation of superfluid turbulence [4].

On the large scales this type of turbulence is commonly described by the two-fluid model. The density of ${ }^{4} \mathrm{He} \rho$ is modeled as a mixture of two fluid components: an inviscid superfluid and a viscous normal fluid, with respective densities $\rho_{\mathrm{s}}$ and $\rho_{\mathrm{n}}$ such that $\rho=\rho_{\mathrm{s}}+\rho_{\mathrm{n}}$. The fluid components are coupled by a mutual friction force [1,3,5,7-9].

Large-scale turbulence in ${ }^{4} \mathrm{He}$ can be generated by various ways. In mechanically driven ${ }^{4} \mathrm{He}$ (so-called "coflow"), the turbulent statistics is similar [10,20,30-32] to that of classical turbulence. In this case both components move in the same direction and the mutual friction force couples them almost at all scales. On the other hand, when a temperature gradient $\nabla T$ is imposed in a channel closed at one end, the heat flux is carried away by the normal fluid with a mean velocity $\boldsymbol{U}_{\mathrm{n}} \propto \nabla T$, while the superfluid component flows in the opposite direction with the mean velocity $\boldsymbol{U}_{\mathrm{s}}$. There is no net mass flow: $\rho_{\mathrm{n}} \boldsymbol{U}_{\mathrm{n}}+\rho_{\mathrm{s}} \boldsymbol{U}_{\mathrm{s}}=0$. The counterflow velocity $\boldsymbol{U}_{\mathrm{ns}}=\boldsymbol{U}_{\mathrm{n}}-\boldsymbol{U}_{\mathrm{s}}$ creates a random vortex tangle with an energy spectrum, peaking at the intervortex scale $\ell$ and with a close to Gaussian statistics, as demonstrated experimentally in Refs. [14-16] and rationalized theoretically in Refs. [18,33]. At large enough $\boldsymbol{U}_{\mathrm{n}}$, the laminar flow of the normal component becomes unstable, creating large-scale turbulence with the energy spectrum dominated by "the outer scale of turbulence" $\Delta \gg \ell$ (e.g., about half-width of the channel). Although the particular mechanisms of the largescale superfluid motion generation are not known in details, recent indirect experimental evidence indicates $[13,33]$ that the large-scale normal-fluid motion gives rise to the superfluid turbulent motion due to the components' coupling by the mutual friction force.

\section{A. Coarse-grained equations for counterflow ${ }^{4} \mathrm{He}$ turbulence}

Our approach $[17,18,34]$ to large-scale counterflow turbulence is based on two Navier-Stokes equations (NSE) for the velocity fluctuations of the normal-fluid and superfluid components $\mathcal{U}_{\mathrm{n}}(\boldsymbol{r}, t)$ and $\mathcal{U}_{\mathrm{s}}(\boldsymbol{r}, t)$. A complication arises from the fact that the counterflow is created in a channel and therefore is, in general, inhomogeneous. However, at large enough Reynolds numbers, the flow in the center of the channel can be approximated as almost space homogeneous [35]. We therefore adopt a simplifying description with space homogeneity and stationarity. Further, we perform the standard Reynolds decomposition of the velocities into their mean and turbulent velocity fluctuations with zero mean:

$$
\begin{aligned}
& \mathcal{U}_{\mathrm{n}}(\boldsymbol{r}, t)=\boldsymbol{U}_{\mathrm{n}}+\boldsymbol{u}_{\mathrm{n}}(\boldsymbol{r}, t), \boldsymbol{U}_{\mathrm{n}}=\left\langle\mathcal{U}_{\mathrm{n}}(\boldsymbol{r}, t)\right\rangle, \\
& \mathcal{U}_{\mathrm{s}}(\boldsymbol{r}, t)=\boldsymbol{U}_{\mathrm{s}}+\boldsymbol{u}_{\mathrm{s}}(\boldsymbol{r}, t), \boldsymbol{U}_{\mathrm{s}}=\left\langle\mathcal{U}_{\mathrm{s}}(\boldsymbol{r}, t) t\right\rangle .
\end{aligned}
$$

The mean velocities are taken below as externally prescribed parameters of the problem. Note that in the classical hydrodynamics, the Navier-Stokes equations are Galilean invariant and one can choose a reference system in which the constant mean velocity vanishes. In the two-fluid counterflow, there is no such reference system and the mean velocities are necessarily present in the equations of motion for the turbulent velocity fluctuations:

$$
\begin{aligned}
& {\left[\frac{\partial}{\partial t}+\left(\boldsymbol{u}_{\mathrm{s}}+\boldsymbol{U}_{\mathrm{s}}\right) \cdot \nabla\right] \boldsymbol{u}_{\mathrm{s}}-\frac{\nabla p_{\mathrm{s}}}{\rho_{\mathrm{s}}}=v_{\mathrm{s}} \Delta \boldsymbol{u}_{\mathrm{s}}+\boldsymbol{f}_{\mathrm{ns}}+\boldsymbol{\varphi}_{\mathrm{s}},} \\
& {\left[\frac{\partial}{\partial t}+\left(\boldsymbol{u}_{\mathrm{n}}+\boldsymbol{U}_{\mathrm{n}}\right) \cdot \nabla\right] \boldsymbol{u}_{\mathrm{n}}-\frac{\nabla p_{\mathrm{n}}}{\rho_{\mathrm{n}}}=v_{\mathrm{n}} \Delta \boldsymbol{u}_{\mathrm{n}}-\frac{\rho_{\mathrm{s}}}{\rho_{\mathrm{n}}} \boldsymbol{f}_{\mathrm{ns}}+\boldsymbol{\varphi}_{\mathrm{n}} .}
\end{aligned}
$$


These equations are coupled by the mutual friction force $[2,6,36] f_{\text {ns }}$. Here $f_{\text {ns }}(r, t)$ is a fluctuating (with zero mean) part of the total mutual friction force $\mathcal{F}_{\mathrm{ns}}(\boldsymbol{r}, t)$ :

$$
\mathcal{F}_{\mathrm{ns}}(\boldsymbol{r}, t)=\boldsymbol{F}_{\mathrm{ns}}+\boldsymbol{f}_{\mathrm{ns}}(\boldsymbol{r}, t) .
$$

The pressures $p_{\mathrm{n}}, p_{\mathrm{s}}$ in Eq. (1b) are given by

$$
\begin{aligned}
p_{\mathrm{n}} & =\frac{\rho_{\mathrm{n}}}{\rho}\left[p+\frac{\rho_{\mathrm{s}} u_{\mathrm{ns}}^{2}}{2}\right], \quad p_{\mathrm{s}}=\frac{\rho_{\mathrm{s}}}{\rho}\left[p-\frac{\rho_{\mathrm{n}} u_{\mathrm{ns}}^{2}}{2}\right], \\
\boldsymbol{u}_{\mathrm{ns}} & =\boldsymbol{u}_{\mathrm{n}}-\boldsymbol{u}_{\mathrm{s}} .
\end{aligned}
$$

The kinematic viscosity of the normal-fluid component is $v_{\mathrm{n}}=\eta / \rho_{\mathrm{n}}$, where $\eta$ is the dynamical viscosity [29] of the normal ${ }^{4} \mathrm{He}$ component. The energy sink [34] in the equation for the superfluid component, with an effective superfluid viscosity [3] $v_{\mathrm{s}}$, accounts for the energy dissipation at the intervortex scale $\ell$ due to vortex reconnections and energy transfer to Kelvin waves. The random forces $\varphi_{\mathrm{s}}$ and $\varphi_{\mathrm{n}}$ represent the forcing of the turbulent flow at large scales.

The physical origin of the mutual friction is the scattering of excitations that constitute the normal fluid on the vortex lines. Any motion of a vortex line relative to the normal-fluid results [3] in a force per unit length of the line, which can be written as

$$
\boldsymbol{f}=-\gamma_{0} \boldsymbol{s}^{\prime} \times \boldsymbol{s}^{\prime} \times\left(\boldsymbol{U}_{\mathrm{ns}}+\boldsymbol{u}_{\mathrm{ns}}\right)+\gamma_{0}^{\prime} \boldsymbol{s}^{\prime} \times\left(\boldsymbol{U}_{\mathrm{ns}}+\boldsymbol{u}_{\mathrm{ns}}\right) .
$$

Here $\boldsymbol{s}^{\prime}$ is a unit vector along the length of the vortex, $\gamma_{0}$ and $\gamma_{0}^{\prime}$ are some phenomenological parameters.

In order to estimate the coarse-grained mutual friction force $f_{\text {ns }}$ in Eq. (1b) one needs to properly average the microscopic Eq. (2) for $f$. The result depends on the statistics of the quantum vortices that in turn depend on the particular turbulent flow properties. In particular, this procedure includes averaging of the force $f$ over directions of the orientations $\boldsymbol{s}^{\prime}$ in the vortex lines. In the relatively simple case of rotating turbulence, the vortices are oriented mostly along the axis of rotation. In this case $\left\langle\boldsymbol{s}^{\prime}\right\rangle$ may be directly related to the direction of the superfluid vorticity $\omega_{\mathrm{s}}:\left\langle\boldsymbol{s}^{\prime}\right\rangle=\omega_{\mathrm{s}} /\left|\omega_{\mathrm{s}}\right|$. The resulting equations were named the "HVBK equations" [5,6] after Hall, Vinen, Bekarevich, and Khalatnikov.

Clearly, the original HVBK equations are not applicable for the superfluid turbulence without global rotation, for which $\left\langle\boldsymbol{s}^{\prime}\right\rangle=0$. In this case, to obtain a coarse-grained representation one should average Eq. (2) over "a physically small volume" of scale $\delta$. This scale should be chosen to be much larger than $\ell$, but still much smaller than the scale $r$ of turbulent fluctuations under consideration, $\ell \ll \delta \ll r$. For such $\delta$, the local line orientations $\boldsymbol{s}^{\prime}$ and $\boldsymbol{s}^{\prime} \otimes \boldsymbol{s}^{\prime}$ in Eq. (2) can be considered as self-averaging in space as they are almost uncorrelated with the $r$-scale fluctuations $\boldsymbol{u}_{\mathrm{ns}}(r)$, which are treated as dynamical variables.

For coflows a number of model expressions were suggested for the fluctuating part of the friction force in the form $f_{\text {ns }} \propto$ $\Omega_{\mathrm{s}} \boldsymbol{u}_{\mathrm{ns}}$. Here the mutual friction frequency $\Omega_{\mathrm{s}}$ was modeled as a dimensional estimate, assuming underlying Kolmogorov energy spectrum for the superfluid component. Examples of such models include $\Omega_{\mathrm{s}}=\alpha\left|\omega_{\mathrm{s}}\right|$ (e.g., in Refs. [32,37,38]), $\Omega_{\mathrm{s}} \alpha \sqrt{\left\langle\omega_{\mathrm{s}}^{2}\right\rangle}$ (e.g., in Refs. $[17,20,39]$ ), and $\Omega_{\mathrm{s}}=\alpha \kappa \mathcal{L}$ (e.g., in Refs. $[2,13,18,20])$. Here $\alpha$ is the dimensionless mutual friction parameter related [1] to $\gamma_{0}$ as $\alpha \rho_{\mathrm{s}} \kappa=\left(1+\alpha^{2}\right) \gamma_{0} ; \mathcal{L}$ is the vortex line density.

In counterflows the dynamics of the vortex tangle is dominated by the stretching of the vortex lines by the counterflow velocity and by their reconnections. Based on experiments in narrow slits, Gorter and Mellink [40] proposed to couple the equations of motion for the components' velocities by the mutual friction force of a phenomenological form $\mathcal{F}_{\mathrm{ns}}=$ $A \rho_{\mathrm{n}} \rho_{\mathrm{s}} \mathcal{U}_{\mathrm{ns}}^{3}$, where $A$ is a temperature-dependent constant. This form was later refined by Vinen [7] for homogeneous turbulence and an isotropic vortex tangle as $\mathcal{F}_{\mathrm{ns}}=G \mathcal{U}_{\mathrm{ns}}$, $G=A \rho_{\mathrm{n}} \rho_{\mathrm{s}} \mathcal{U}_{\mathrm{ns}}^{2}$. Taking into account the relation between the vortex line density and the counterflow velocity in the steadystate isotropic tangle, it can be further rewritten [2] as $\mathcal{F}_{\mathrm{ns}}=$ $\frac{2}{3} \alpha \kappa \mathcal{L} \mathcal{U}_{\mathrm{ns}}$.

The tangle anisotropy with respect to the direction of $\boldsymbol{U}_{\mathrm{ns}}$ can be described by the Schwarz's indices [9]:

$$
\begin{aligned}
& I_{\|}=\frac{1}{L_{\mathrm{tot}}} \int_{\mathcal{C}}\left[1-\left(\mathbf{s}^{\prime} \cdot \hat{\boldsymbol{r}}_{\|}\right)^{2}\right] d \xi=\left\langle\left[1-\left(\mathbf{s}^{\prime} \cdot \hat{\boldsymbol{r}}_{\|}\right)^{2}\right\rangle_{\mathcal{C}},\right. \\
& I_{\perp}=\frac{1}{L_{\mathrm{tot}}} \int_{\mathcal{C}}\left[1-\left(\mathbf{s}^{\prime} \cdot \hat{r}_{\perp}\right)^{2}\right] d \xi=\left\langle\left[1-\left(\mathbf{s}^{\prime} \cdot \hat{\mathbf{r}}_{\perp}\right)^{2}\right\rangle_{\mathcal{C}} .\right.
\end{aligned}
$$

Here $L_{\text {tot }}$ is the total vortex length in the whole vortex configuration $\mathcal{C}$ over which integrals are taken and $\hat{\boldsymbol{r}}_{\|}$and $\hat{\boldsymbol{r}}_{\perp}$ are unit vectors in the directions parallel and perpendicular to $\boldsymbol{U}_{\mathrm{ns}}$, respectively. Using (3a) and (3b), the mutual friction force may be written as

$$
\begin{gathered}
\boldsymbol{F}_{\mathrm{ns}}=\alpha \kappa \mathcal{L} I_{\|} \boldsymbol{U}_{\mathrm{ns}}, \\
\boldsymbol{f}_{\mathrm{ns}}(\boldsymbol{r}, t)=\alpha \kappa \mathcal{L}\left[I_{\|} \boldsymbol{u}_{\mathrm{ns}}^{\|}(\boldsymbol{r}, t)+I_{\perp} \boldsymbol{u}_{\mathrm{ns}}^{\perp}(\boldsymbol{r}, t)\right] .
\end{gathered}
$$

Notably, the second nondissipative term in Eq. (2) $\propto \gamma_{0}^{\prime}$ vanishes by symmetry and does not contribute [9] to the averaged quantity $\mathcal{F}_{\text {ns }}$. The mean mutual friction force $\boldsymbol{F}_{\text {ns }}$ (3c), found earlier by Schwarz [9], enters into the equations for the mean velocities $\boldsymbol{U}_{\mathrm{n}}$ and $\boldsymbol{U}_{\mathrm{s}}$, which we do not discuss. The fluctuating part (3d) of the mutual friction force $\boldsymbol{f}_{\mathrm{ns}}(\boldsymbol{r}, t)$ enters Eq. (1b). The vector components of turbulent counterflow velocity fluctuations $\boldsymbol{u}_{\mathrm{ns}}^{\|}$and $\boldsymbol{u}_{\mathrm{ns}}^{\perp}$ are oriented in the directions of $\hat{\boldsymbol{r}}_{\|}$and $\hat{\boldsymbol{r}}_{\perp}$, respectively.

The definitions Eq. (3) do not take into account that the turbulent intensity of the normal-fluid velocity $\sqrt{\left\langle\left|\boldsymbol{u}_{\mathrm{n}}\right|^{2}\right\rangle} / U_{\mathrm{ns}}$ in the counterflow turbulence is not very small and can reach [12] values of about 0.25 . So, strictly speaking, in Eqs. (3a) and (3b) the directions $\hat{\boldsymbol{r}}_{\|}$and $\hat{\boldsymbol{r}}_{\perp}$, should be taken along and orthogonal to the total counterflow velocity $\mathcal{U}_{\text {ns }}$. In addition, one should take into account the space-time dependence of $\mathcal{L}$ in Eq. (3d). However, since the leading contribution to $\sqrt{\left\langle\left|\boldsymbol{u}_{\mathrm{ns}}\right|^{2}\right\rangle}$ originates from the outer scale of turbulence $\Delta \gg$ $\ell$, for the motions of the scale $\ell$ this correction effectively adds to $U_{\mathrm{ns}}$ and we can neglect the influence of the velocity fluctuations of the scale $\boldsymbol{r}$ on the vortex line density, replace an average of the product by the product of averages and consider $\mathcal{L}$ as constant. 
The equation (3d) may be identically rewritten as

$$
\begin{gathered}
\boldsymbol{f}_{\mathrm{ns}}(\boldsymbol{r}, t)=\Omega_{\mathrm{s}}\left[\boldsymbol{u}_{\mathrm{ns}}+\frac{I_{\|}-I_{\perp}}{2}\left(2 \boldsymbol{u}_{\mathrm{ns}}^{\|}-\boldsymbol{u}_{\mathrm{ns}}^{\perp}\right)\right], \\
\Omega_{\mathrm{s}}=\frac{2}{3} \alpha \kappa \mathcal{L}, \quad \boldsymbol{u}_{\mathrm{ns}}=\boldsymbol{u}_{\mathrm{ns}}^{\|}+\boldsymbol{u}_{\mathrm{ns}}^{\perp} .
\end{gathered}
$$

Taking the numerical values of $I_{\|}$and $I_{\perp}$ (see, e.g., Table IV for $T=1.6 \mathrm{~K}$ in Ref. [41]) we see that $\left(I_{\perp}-I_{\|}\right) / 2 \approx 0.05$. This means that with a reasonable accuracy of about $5-10 \%$ we can neglect the anisotropy term in Eq. (4) and use the simple form $\boldsymbol{f}_{\mathrm{ns}}(\boldsymbol{r}, t)=\Omega_{\mathrm{s}} \boldsymbol{u}_{\mathrm{ns}}(\boldsymbol{r}, t)$ as a good approximation for $\boldsymbol{f}_{\mathrm{ns}}$ even for counterflow turbulence. Furthermore, in this paper we do not consider the dependence of $\mathcal{L}$ on the flow parameters and use the mutual friction frequency $\Omega_{\mathrm{s}}$ as a prescribed external control parameter. It can be estimated or measured for each particular flow conditions.

\section{B. Statistical characteristics of anisotropic turbulence}

\section{Velocity correlation function}

A useful characterization of homogeneous superfluid ${ }^{4} \mathrm{He}$ turbulence is furnished by the three-dimensional (3D) correlation functions of the normal and superfluid turbulent velocity fluctuations in $\boldsymbol{k}$ representation:

$$
\begin{array}{r}
(2 \pi)^{3} \delta\left(\boldsymbol{k}-\boldsymbol{k}^{\prime}\right) \mathcal{E}_{i j}^{\alpha \beta}(\boldsymbol{k})=\left\langle v_{i}^{\alpha}(\boldsymbol{k}) v_{j}^{* \beta}\left(\boldsymbol{k}^{\prime}\right)\right\rangle, \\
\mathcal{E}_{j}^{\alpha \beta}(\boldsymbol{k})=\mathcal{E}_{j j}^{\alpha \beta}(\boldsymbol{k}), \quad \mathcal{E}_{i j}(\boldsymbol{k}) \equiv \sum_{\alpha=x, y, z} \mathcal{E}_{i j}^{\alpha \alpha}(\boldsymbol{k}) .
\end{array}
$$

Here $\delta\left(\boldsymbol{k}-\boldsymbol{k}^{\prime}\right)$ is a 3D Dirac's delta function, $\boldsymbol{v}_{j}(\boldsymbol{k})$ is the Fourier transform of $\boldsymbol{u}_{j}(\boldsymbol{r})$,

$$
\begin{aligned}
& \boldsymbol{v}_{j}(\boldsymbol{k})=\int \boldsymbol{u}_{j}(\boldsymbol{r}) \exp (-i \boldsymbol{k} \cdot \boldsymbol{r}) d \boldsymbol{r} \\
& \boldsymbol{u}_{j}(\boldsymbol{r})=\int \boldsymbol{v}_{j}(\boldsymbol{k}) \exp (i \boldsymbol{k} \cdot \boldsymbol{r}) \frac{d \boldsymbol{k}}{(2 \pi)^{3}}
\end{aligned}
$$

the indices $\alpha, \beta=\{x, y, z\}$ denote Cartesian coordinates, the subscripts " $i, j$ " denote the normal $(i, j=n)$ or the superfluid $(i, j=s)$ fluid components and ${ }^{*}$ stands for complex conjugation. In the rest of the paper, we denote the trace of any tensor according to $\mathcal{E}_{i j}(\boldsymbol{k})=\sum_{\alpha} \mathcal{E}_{i j}^{\alpha \alpha}(\boldsymbol{k})$. The correlation function $\mathcal{E}_{i j}^{\alpha \beta}(\boldsymbol{k})$ and the Fourier transform $(5 \mathrm{c})$ are defined such that the kinetic energy density per unit mass $E_{j}$ reads

$$
E_{j}=\frac{1}{2}\left\langle\left|\boldsymbol{u}_{j}(\boldsymbol{r})\right|^{2}\right\rangle=\frac{1}{2} \int \mathcal{E}_{j j}(\boldsymbol{k}) \frac{d^{3} k}{(2 \pi)^{3}} .
$$

The dimensionality of the energy density is $\left[E_{j j}\right]=\mathrm{cm}^{2} / \mathrm{s}^{2}$, while the dimensionality of the $3 \mathrm{D}$ energy spectra is $\left[\mathcal{E}_{j j}\right]=$ $\mathrm{cm}^{5} / \mathrm{s}^{2}$.

Due to the presence of a preferred direction (the counterflow velocity), the resulting turbulence has an axial symmetry around that direction. Accordingly, $\mathcal{E}_{i j}^{\alpha \beta}(\boldsymbol{k})$ depends only on two projections $k_{\|}$and $k_{\perp}$ of the wave vector $\boldsymbol{k}: \boldsymbol{k}_{\|} \equiv \boldsymbol{U}_{\mathrm{ns}}(\boldsymbol{k}$. $\left.\boldsymbol{U}_{\mathrm{ns}}\right) / U_{\mathrm{ns}}^{2}$ and $\boldsymbol{k}_{\perp} \perp \boldsymbol{U}_{\mathrm{ns}}$, being independent of the angle $\varphi$ in the $\perp$ plane, orthogonal to $\boldsymbol{U}_{\mathrm{ns}}: \mathcal{E}_{i j}^{\alpha \beta}(\boldsymbol{k}) \Rightarrow \mathcal{E}_{i j}^{\alpha \beta}\left(k_{\|}, k_{\perp}\right)$. This allows us to define a $2 \mathrm{D}$ object $E_{i j}^{\alpha \beta}\left(k_{\|}, k_{\perp}\right)$ that still contains all the information about second-order statistics of the counterflow turbulence:

$$
E_{i j}^{\alpha \beta}\left(k_{\|}, k_{\perp}\right) \equiv \frac{k_{\perp}}{4 \pi^{2}} \mathcal{E}_{i j}^{\alpha \beta}\left(k_{\|}, k_{\perp}\right)
$$

Another way to represent the same information is to introduce a polar angle $\theta=\angle\left(\boldsymbol{k}, \boldsymbol{U}_{\mathrm{ns}}\right)$, to represent the wave-vector length as $k=\sqrt{k_{\|}^{2}+k_{\perp}^{2}}$ and to define a 2D object $\tilde{E}_{i j}^{\alpha \beta}(k, \theta)$ in spherical coordinates:

$$
\tilde{E}_{i j}^{\alpha \beta}(k, \theta) \equiv \frac{k \mathcal{E}_{i j}^{\alpha \beta}(\boldsymbol{k})}{4 \pi^{2}}=\frac{k}{4 \pi^{2}} \mathcal{E}_{i j}^{\alpha \beta}(k \cos \theta, k \sin \theta) .
$$

The dimensionality of 2D energy spectra and correlation functions is $\left[E_{i j}^{\alpha \beta}\right]=\left[\tilde{E}_{i j}^{\alpha \beta}\right]=\mathrm{cm}^{4} / \mathrm{s}^{2}$.

A more compact but less detailed information on the statistics of turbulence is provided by a set of one-dimensional (1D) energy spectra. The most traditional are the 1D "spherical" energy spectra and the cross-correlation function ${ }^{\bullet} E_{i j}^{\alpha \beta}(k)$, averaged over a spherical surface of radius $k$ :

$$
\begin{aligned}
\bullet^{\bullet} E_{i j}^{\alpha \beta}(k) & =\int \mathcal{E}_{i j}^{\alpha \beta}\left(k_{\|}, k_{\perp}\right) \delta\left(k-\sqrt{k_{\|}^{2}+k_{\perp}^{2}}\right) \frac{k_{\perp} d k_{\perp} d k_{\|}}{4 \pi^{2}} \\
& =\int E_{i j}^{\alpha \beta}\left(k_{\|}, k_{\perp}\right) \delta\left(k-\sqrt{k_{\|}^{2}+k_{\perp}^{2}}\right) d k_{\perp} d k_{\|} \\
& =k \int_{-1}^{1} \tilde{E}_{i j}^{\alpha \beta}(k, \theta) d \cos \theta .
\end{aligned}
$$

In the isotropic case, when $\mathcal{E}_{i j}^{\alpha \beta}$ depends only on $k=$ $\sqrt{k_{\|}^{2}+k_{\perp}^{2}}$, this representation simplifies to a well-known relationship:

$$
{ }^{\bullet} E_{i j}^{\alpha \beta}(k)=\frac{k^{2}}{2 \pi^{2}} \mathcal{E}_{i j}^{\alpha \beta}(k), \text { for spherical symmetry. }
$$

Further information about the anisotropy of the secondorder statistics is obtained by comparing the spherical 1D spectra $E_{i j}(k)$ with a set of $1 \mathrm{D}$ spectra averaged differently. A natural choice are spectra averaged over a cylinder of radius $k_{\perp}$ with the axis oriented along $\boldsymbol{k}_{\|}$. This results in the cylindrical 1D spectra,

$$
{ }^{\circ} E_{i j}^{\alpha \beta}\left(k_{\perp}\right)=\int E_{i j}^{\alpha \beta}\left(k_{\|}, k_{\perp}\right) d k_{\|} .
$$

Alternatively, one can average the $3 \mathrm{D}$ function $\mathcal{E}_{i j}\left(k_{\|}, k_{\perp}\right)$ over a plane. Here we choose two planes:

(1) $1 \mathrm{D}$ spectra ${ }^{\perp} E_{i j}^{\alpha \beta}\left(k_{\|}\right)$, averaged over a $\perp$ plane, orthogonal to $\boldsymbol{U}_{\mathrm{ns}}$,

$$
{ }^{\perp} E_{i j}^{\alpha \beta}\left(k_{\|}\right)=\int E_{i j}^{\alpha \beta}\left(k_{\|}, k_{\perp}\right) d k_{\perp} .
$$

These spectra depend on the streamwise projection $k_{\|}$of the wave vector $\boldsymbol{k}$.

(2) $1 \mathrm{D}$ spectra $\| E_{i j}^{\alpha \beta}\left(k_{\perp}\right)$, averaged over the \| plane, oriented along $\boldsymbol{U}_{\mathrm{ns}}$. We chose for concreteness the plane $\left(k_{x}, k_{z}\right)$, such that $k_{\|}=k_{x}$ and the spectra depend on $k_{y}$ :

$$
{ }^{\|} E_{i j}^{\alpha \beta}\left(k_{y}\right)=\int \mathcal{E}_{i j}^{\alpha \beta}\left(k_{\|}, \sqrt{k_{z}^{2}+k_{y}^{2}}\right) \frac{d k_{\|} d k_{z}}{4 \pi^{2}} .
$$


Note that the 2D- and 1D-energy spectra are defined such that the kinetic energy density per unit mass $\mathcal{E}_{j}$ can be found as

$$
\begin{aligned}
2 E_{j} & =\int \mathcal{E}_{j j}(\boldsymbol{k}) \frac{d^{3} k}{(2 \pi)^{3}}=\int E_{j j}\left(k_{\|}, k_{\perp}\right) d k_{\|} d k_{\perp} \\
& =\int \tilde{E}_{j j}^{\alpha \beta}(k, \theta) k d k d \cos \theta=\int{ }^{\alpha \beta} E_{j j}^{\alpha \beta}(k) d k \\
& =\int{ }^{\perp} E_{j j}^{\alpha \beta}\left(k_{\|}\right) d k_{\|}=\int{ }^{\|} E_{j j}^{\alpha \beta}\left(k_{y}\right) d k_{y} .
\end{aligned}
$$

The tensor structure of the energy spectra will be considered only in Sec. IIID. In the rest of Sec. III we will restrict ourselves by discussing only scalar versions of the energy spectra, which are the traces of their tensorial counterparts.

\section{Velocity structure functions}

Another presentation of the statistics of turbulence is provided by the second-order velocity structure functions,

$$
\begin{aligned}
\delta_{R} u_{j}^{\alpha} & \equiv u_{j}^{\alpha}(\boldsymbol{R}+\boldsymbol{r}, t)-u_{j}^{\alpha}(\boldsymbol{r}, t), \\
S_{j}^{\alpha \beta}(\boldsymbol{R}) & \equiv\left\langle\delta_{R} u_{j}^{\alpha} \delta_{R} u_{j}^{\beta}\right\rangle .
\end{aligned}
$$

The trace $S_{j}(\boldsymbol{R}) \equiv \sum_{\alpha} S_{j}^{\alpha \alpha}(\boldsymbol{R})$ is a measure of the kinetic energy of turbulent (normal or superfluid) velocity fluctuations on scale $R$. Recently, the streamwise normal velocity across a channel $v^{x}(y, t)$ was measured using thin lines of the triplet-state $\mathrm{He}_{2}$ molecular tracers created by a femtosecondlaser field ionization of $\mathrm{He}$ atoms [42] across the channel. This way, the transversal second-order structure functions $[11,12]$ of the normal-fluid velocity differences $S_{\mathrm{n}}^{x x}\left(R_{y}\right)$ were obtained. Similarly, one can use two or more tracer lines, separated in the stream-line direction $\widehat{\boldsymbol{x}}$, to measure the longitudinal structure function $S_{\mathrm{n}}^{x x}\left(R_{x}\right)$ and even inclined structure function $S_{\mathrm{n}}^{x x}\left(R_{x}, R_{y}\right)$.

Using the definition of the structure functions (9a) and the one-dimensional version of the inverse Fourier transform (5d) one gets

$$
\begin{aligned}
& S_{j}^{x x}\left(R_{y}\right)=8 \int_{0}^{\infty}{ }^{\|} E_{j}^{x x}\left(k_{y}\right) \sin ^{2} \frac{k_{y} R_{y}}{2} d k_{y}, \\
& S_{j}^{x x}\left(R_{x}\right)=8 \int_{0}^{\infty}{ }^{\perp} E_{\mathrm{n}}^{x x}\left(k_{x}\right) \sin ^{2} \frac{k_{x} R_{x}}{2} d k_{x} .
\end{aligned}
$$

Analyzing the integrals (10) for the scale-invariant spectra $E(k) \propto k^{-m}$ one concludes that they converge in the window of locality,

$$
1<m<3 .
$$

In this window, the leading contribution to the integrals (10) comes from the region $k R \sim 1$ and

$$
S_{j}(R) \propto R^{n}, \quad n=m-1 .
$$

This is a well-known relationship. For example, $n=2 / 3$ for the K41 spectrum with $m=5 / 3$ [which satisfy (11a)]. However, for fast decaying spectra with $m \geqslant 3$ the integrals (10a) diverge in the infrared region $k R \ll 1$ with the main contribution coming from the energy containing region $k \sim k_{0}$, giving

$$
S_{j}^{\alpha \alpha}(R) \propto R^{2} .
$$

We see that connection between $m$ and $n$ for fast decaying spectra with $m>3$ is lost. To recover it for $m>3$ we consider structure functions of the velocity second differences [43,44],

$$
\begin{aligned}
\Delta_{R} u_{j}^{\alpha} & \equiv u_{j}^{\alpha}(2 R+\boldsymbol{r}, t)-2 u_{j}^{\alpha}(\boldsymbol{R}+\boldsymbol{r}, t)+u_{j}^{\alpha}(\boldsymbol{r}, t), \\
\widetilde{S}_{j}^{\alpha \beta}(\boldsymbol{R}) & \equiv\left\langle\Delta_{R} u_{j}^{\alpha} \Delta_{R} u_{j}^{\beta}\right\rangle .
\end{aligned}
$$

Now instead of Eq. (10) we have

$$
\begin{aligned}
& \widetilde{S}_{j}^{x x}\left(R_{y}\right)=32 \int_{0}^{\infty}{ }^{{ }} E_{j}^{x x}\left(k_{y}\right) \sin ^{4} \frac{k_{y} R_{y}}{2} d k_{y}, \\
& \tilde{S}_{j}^{x x}\left(R_{x}\right)=32 \int_{0}^{\infty}{ }^{\perp} E_{j}^{x x}\left(k_{x}\right) \sin ^{4} \frac{k_{x} R_{x}}{2} d k_{x} .
\end{aligned}
$$

Now these integrals converge in the extended window of locality,

$$
1<m<5 .
$$

In this window, the leading contribution to the integrals (13) again comes from the region $k R \sim 1$ and, similarly to Eq. (11b), for the scale-invariant spectrum $E(k) \propto k^{-m}$ we have

$$
\widetilde{S}_{j}^{\alpha \alpha}(R) \propto R^{n}, \quad n=m-1 .
$$

For $m>5$ the integrals (13) diverge in the infrared region and

$$
\tilde{S}_{j}^{\alpha \alpha}(R) \propto R^{4} .
$$

It is worth noting that the relations (11b) and (14b) are valid in the limit of the infinite inertial interval. For a finite inertial interval which is typical for the experimental conditions, the structure functions have a complicated functional dependence, mixing the inertial and viscous behavior and the original scaling of the energy spectra is reproduced over very short intervals of scales [13]. Nevertheless, when experimental conditions do not allow one to measure the energy spectra directly, the structure functions remain the preferred tool to access the statistics of the velocity fluctuations. In a turbulent counterflow, where the energy spectra are not scale invariant, the quantitative analysis of the structure functions may not be meaningful. Nevertheless a qualitative difference between structure functions measured along different directions may confirm the presence of the spectral anisotropy.

\section{Physical origin of the strong anisotropy of counterflow turbulence}

In a recent Letter [19] it was shown that the energy spectra in counterflow turbulence are expected to be strongly anisotropic. To keep the present paper self-contained, we repeat here some of that discussion and add further clarifications to the analysis. 
We start with a balance equation $[18,19]$ for the 2D-energy spectrum $\tilde{E}_{j}(k, \theta)$ in the counterflow turbulence with axial symmetry:

$$
\begin{aligned}
\frac{\partial \tilde{E}_{j}(k, \theta, t)}{\partial t}+\operatorname{div}_{\boldsymbol{k}}\left[\boldsymbol{\varepsilon}_{j}(\boldsymbol{k})\right] & =-\mathcal{D}_{j}^{\mathrm{mf}}(k, \theta)-\mathcal{D}_{j}^{v}(k, \theta), \\
\mathcal{D}_{j}^{\mathrm{mf}}(k, \theta) & =\Omega_{j}\left[\tilde{E}_{j}(k, \theta)-\tilde{E}_{\mathrm{ns}}(k, \theta)\right], \\
\mathcal{D}_{j}^{v}(k, \theta) & =2 v_{j} k^{2} \tilde{E}_{j}(k, \theta) .
\end{aligned}
$$

Here $\operatorname{div}_{k}\left[\boldsymbol{\varepsilon}_{j}(\boldsymbol{k})\right]$ is the transfer term due to inertial nonlinear effects. The terms on the right-hand side describe the energy dissipation rate due to the mutual friction $\mathcal{D}_{j}^{\mathrm{mf}}(k, \theta)$ and due to the viscous effects $\mathcal{D}_{j}^{v}(k, \theta)$. To keep the presentation concise, we introduced a notation $\Omega_{\mathrm{n}}=\Omega_{\mathrm{s}} \rho_{\mathrm{s}} / \rho_{\mathrm{n}}$.

The origin of the energy spectra anisotropy in counterflow turbulence can be deduced from the form of the dissipation rate $\mathcal{D}_{j}^{\mathrm{mf}}(k, \theta)(15 \mathrm{~b})$. In this term, the cross-correlation function $\tilde{E}_{\mathrm{ns}}(k, \theta)$ has the following form [cf. Eq. (13) in Ref. [17]]:

$$
\tilde{E}_{\mathrm{ns}}(k, \theta)=\frac{A B}{B^{2}+\left(\boldsymbol{k} \cdot \boldsymbol{U}_{\mathrm{ns}}\right)^{2}} .
$$

Here $A=\Omega_{\mathrm{s}} \tilde{E}_{\mathrm{n}}(k, \theta)+\Omega_{\mathrm{n}} \tilde{E}_{\mathrm{s}}(k, \theta)$ and $B$ can be written [18] as $B=\Omega_{\mathrm{ns}}=\Omega_{\mathrm{n}}+\Omega_{\mathrm{s}}$. We further note [18,19] that when two components are highly correlated, the cross-correlation may be accurately represented by the corresponding energy spectra. For wave numbers where the components are not correlated, $\tilde{E}_{\mathrm{ns}}(k, \theta)$ is small and the accuracy of its representation is less important. This allows us to decouple $\tilde{E}_{\mathrm{ns}}(k, \theta)$ in Eq. (15) for each component as follows:

$$
\begin{aligned}
\tilde{E}_{\mathrm{ns}}(k, \theta) & =\tilde{E}_{j}(k, \theta) D(k, \theta), \\
D(k, \theta) & =1 /\left[1+\left(\frac{k U_{\mathrm{ns}} \cos \theta}{\Omega_{\mathrm{ns}}}\right)^{2}\right] .
\end{aligned}
$$

Note that averaging Eq. (16c) over $\theta$ results in the equation for $D(k)$, used in the theory of isotropic counterflow turbulence [18]:

$$
\begin{aligned}
D(k) & \equiv\langle D(k, \theta)\rangle_{\theta}=\int_{0}^{1} D(k, \theta) d \cos \theta \\
& =\frac{k_{\times}}{k} \arctan \frac{k}{k_{\times}}, \quad k_{\times} \equiv \frac{\Omega_{\mathrm{ns}}}{U_{\mathrm{ns}}} .
\end{aligned}
$$

The function $D(k, \theta)$ in Eq. (16) describes the level of decorrelation of the normal-fluid and superfluid velocity components by the counterflow velocity. Within the approximation $(16 \mathrm{~b}), D(k, \theta)$ defines the rate of energy dissipation caused by mutual friction:

$$
\mathcal{D}_{j}^{\mathrm{mf}}(k, \theta)=\Omega_{j} \tilde{E}_{j}[1-D(k, \theta)] .
$$

For small $k$ or even for large $k$ with $\boldsymbol{k}$ almost perpendicular to $\boldsymbol{U}_{\text {ns }}$ (i.e., $\cos \theta \ll 1$ ), $D(k, \theta) \simeq 1$, the normal and superfluid velocities are almost fully coupled, and the rate of the energy dissipation $\mathcal{D}_{j}^{\mathrm{mf}}(k, \theta) \ll \Omega_{j}$ is small. In this case, the role of mutual friction is minor and we expect the energy spectrum $\tilde{E}_{j}(k, \theta)$ to be close to the classical prediction $E_{\mathrm{K} 41}(k) \propto k^{-5 / 3}$. For large $k$ and for $\boldsymbol{k}$ with $\cos \theta \sim 1$, $D(k, \theta) \ll 1$, the velocity components are almost decoupled, $\mathcal{D}_{j}(k, \theta) \ll 1$ and the mutual-friction energy dissipation is maximal: $\mathcal{D}_{j}^{\mathrm{mf}}(k, \theta) \approx \Omega_{j}$. This situation is similar to that in ${ }^{3} \mathrm{He}$ with the normal-fluid component at rest, for which $\mathcal{D}_{\mathrm{s}}^{\mathrm{mf}}(k, \theta)=\Omega_{\mathrm{s}}$. In this case, we can expect that the energy dissipation by mutual friction strongly suppresses the energy spectra, much below the $\mathrm{K} 41$ expectation $E_{\mathrm{K} 41}(k)$ up to the level typical for the ${ }^{3} \mathrm{He}$ turbulence $[21,22,36,45]$. Next, we note that $\Omega_{\mathrm{ns}}=\Omega_{\mathrm{s}}+\Omega_{\mathrm{n}}=\rho \Omega_{\mathrm{s}} / \rho_{\mathrm{n}}$. Then Eq. (16c) may be rewritten as

$$
D(k, \theta)=1 /\left[1+\left(\frac{\rho_{\mathrm{n}} k U_{\mathrm{ns}} \cos \theta}{\rho \Omega_{\mathrm{s}}}\right)^{2}\right] .
$$

At low temperatures $\rho_{\mathrm{n}} / \rho$ is small and the velocity decorrelation is considerable only at large $k$, while at higher temperature $\rho_{\mathrm{n}} / \rho \approx 1$ and the energy dissipation by mutual friction is effective at all scales.

Combining all these considerations, we expect the energy spectra $\tilde{E}_{j}(k, \cos \theta)$ to become more anisotropic with increasing $k$, with most of the energy concentrated in the range of small $\cos \theta$, i.e., in the wave-vector plane orthogonal to the counterflow velocity $\boldsymbol{U}_{\mathrm{ns}}$. This effect is milder at low $T$ and stronger at higher temperatures.

\section{STRONG ANISOTROPY OF ENERGY SPECTRA}

\section{A. Simulation parameters and numerical procedure}

The direct numerical simulation of the coupled Eq. (1b) were carried out using a fully de-aliased pseudospectral code with a resolution of $256^{3}$ collocation points in a triply periodic domain of size $L=2 \pi$. The parameters of the simulations are summarized in Table I. To obtain the steady-state evolution, velocity fields of the normal and superfluid components are stirred by two independent isotropic random Gaussian forcings:

$$
\left\langle\tilde{\boldsymbol{\varphi}}_{u}(\boldsymbol{k}, t) \cdot \tilde{\boldsymbol{\varphi}}_{u}^{*}\left(\boldsymbol{k}^{\prime}, t^{\prime}\right)\right\rangle=\Phi(k) \delta\left(\boldsymbol{k}-\boldsymbol{k}^{\prime}\right) \delta\left(t-t^{\prime}\right) \widehat{P}(\boldsymbol{k}),
$$

where $\widehat{P}(\boldsymbol{k})$ is a projector assuring incompressibility and $\Phi(k)=\Phi_{0} k^{-3}$; the forcing amplitude $\Phi_{0}$ is nonzero only in a given band of Fourier modes: $k \in[0.5,1.5]$. Both components are forced with the same amplitude to allow direct comparison with simulations of the uncoupled equations. The time integration is performed using a second-order AdamsBashforth scheme with the viscous term exactly integrated. Simulations for all temperatures were carried out with the normal-fluid viscosity fixed at $\tilde{v}_{\mathrm{n}}=0.003$ and the value of $\tilde{v}_{\mathrm{s}}$ is found using the known value of ratio $v_{\mathrm{s}} / v_{\mathrm{n}}$ at each temperature.

To properly expose various aspects of the counterflow turbulence statistics we chose several sets of governing parameters for the simulations. Since the material parameters of ${ }^{4} \mathrm{He}$ are strongly temperature dependent [29] (see Table I, columns 3-5), we consider three temperatures, corresponding to an experimentally accessible range $T=1.65,1.85$, and $2.0 \mathrm{~K}$. At low temperatures the superfluid component is dominant and has a lower viscosity, while at high $T$ the density of the normal-fluid component is larger, while its kinematic viscosity is lower. At $T=1.85 \mathrm{~K}$, the densities and the viscosities of two components are closely matched. 
TABLE I. Parameters of simulations by columns: (1) Run No.; (2) temperature (K); (3 and 4) ratios of the superfluid and normal-fluid densities [29], $\rho_{\mathrm{s}} / \rho_{\mathrm{n}}$ and viscosities $v_{\mathrm{s}} / \nu_{\mathrm{n}}$; (5) the mutual friction parameter $\alpha$; (6 and 7) the numerical values of the kinematic viscosity of the normal-fluid and superfluid components $\tilde{v}_{\mathrm{n}}$ and $\tilde{v}_{\mathrm{s}} ;(8$ and 9) the numerical values of mutual friction frequency $\Omega$ and counterflow velocity $V$; (10 and 11) the root mean square (rms) normal-fluid and superfluid turbulent velocity fluctuations $v_{\mathrm{T}}^{\mathrm{n}}$ and $v_{\mathrm{T}}^{\mathrm{s}}$; (12 and 13) the normal-fluid and superfluid Reynolds numbers; (14) $k_{\times}$, Eq. (16d). For details see Sec. III A. In all simulations, the number of collocation points along each axis is $N=256$; the computational box size is $L=2 \pi$; the range of forced wave numbers $k^{\tilde{\varphi}}=[0.5,1.5]$.

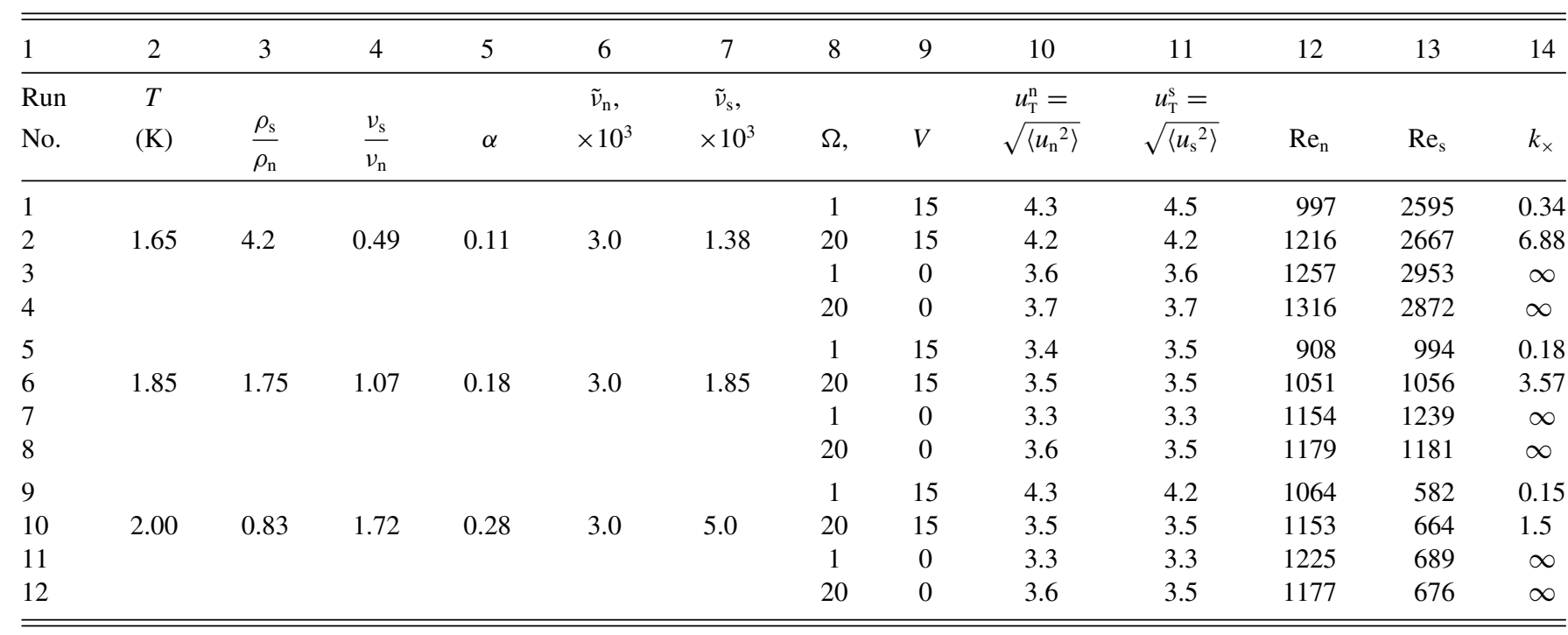

As was shown in previous studies $[17,18,33]$, the major role in the statistics of the counterflow in superfluid ${ }^{4} \mathrm{He}$ is played by the ratio of the mutual friction frequency and the counterflow Doppler frequency $\Omega_{\mathrm{ns}} /\left(k U_{\mathrm{ns}}\right)$ [cf. Eq. (16d)]. To explore various scenarios, we use one counterflow velocity $U_{\mathrm{ns}}$ and two very different values of $\Omega_{\mathrm{s}}$. To emphasize the importance of the flow anisotropy, we compare the results for the counterflow with simulations of coflow $U_{\mathrm{ns}}=0$, keeping the rest of the parameters unchanged. The detailed study of the statistics of the coflow was reported in Ref. [20]. Other parameters of the simulations were chosen based on dimensionless numbers: (i) the Reynolds numbers $\operatorname{Re}_{j}=\frac{\Delta u}{v_{j} k_{0}}$, (ii) the turbulent intensity $\frac{U_{\mathrm{ns}}}{\Delta u}$, and (iii) the dimensionless crossover scale $q_{\times}=\frac{\Omega_{\mathrm{ns}}}{k_{0} U_{\mathrm{ns}}}=\frac{k_{\times}}{k_{0}}$. Here $\Delta u$ is the root-mean square (rms) of the normal-fluid turbulent velocity fluctuations, and $k_{0}=1$ is the outer scale of turbulence. The numerical values of the dimensionless counterflow velocity $V=15$ and the mutual friction frequency $\Omega=1$ and 20 are listed in Table I, columns 8 and 9 . In this way, for each temperature we have four runs, labeled below as $V_{0} \Omega_{1}, V_{0} \Omega_{20}, V_{15} \Omega_{1}$, and $V_{15} \Omega_{20}$. These values of the counterflow velocity and the mutual friction frequency are similar to the experimental flow parameters $[13,18]$, although they do not match any particular experimental conditions. Moreover, the values of the wave numbers corresponding to the intervortex distance estimated using Eq. (4) $k_{\ell} \approx 10^{3}-6 \times 10^{3}$ are of the same order as the intervortex scale in the experiments of Ref. [13], confirming the applicability of the large-scale description. Reference simulations of the uncoupled Eq. (1a) with $\Omega_{\mathrm{s}}=0, U_{\mathrm{ns}}=0$, representing classical hydrodynamic turbulence for the same parameters of the flow, are labeled $\mathrm{Cl}$.

The correlation between components in the counterflow becomes gradually weaker with increasing $\cos \theta$ for
$T=1.65 \mathrm{~K}$, while for $T=2.0 \mathrm{~K}$ the normal fluid and superfluid are essentially uncorrelated for $\cos \theta \gtrsim 0.1$.

\section{B. Spherical energy spectra ${ }^{\bullet} E_{j j}(k)$ and cross-correlations ${ }^{\bullet} E_{\mathrm{ns}}(k)$}

The energy spectra are influenced by a few competing factors: the viscous dissipation, the dissipation by mutual friction, and the decoupling due to counterflow velocity. To find their relative importance, we first ignore the present angular dependence and consider the spherically averaged spectrum ${ }^{\bullet} E_{j}(k)$ and the normalized cross-correlation function:

$$
\bullet R(k)=\frac{2^{\bullet} E_{\mathrm{ns}}(k)}{\bullet_{\mathrm{nn}}(k)+{ }^{\bullet} E_{\mathrm{ss}}(k)} .
$$

In the upper row of Fig. 1 we plot the spectra ${ }^{\bullet} E_{j}(k)$, compensated by the classical scaling $k^{5 / 3}$ for different flow conditions. In the lower row we show the cross-correlations Eq. (20). In this figure and in Figs. 2-7 the results for $T=1.65 \mathrm{~K}$ are shown in the left column [(a) and (d)], for $T=1.85 \mathrm{~K}$ in the middle column [(b) and (e)], and for $T=2.0 \mathrm{~K}$ in the right column [(c) and (f)]. The effect of viscous dissipation is clearly seen in the spectra of the uncoupled components, corresponding to classical turbulence and marked "Cl", black lines. The spectra almost coincide for $T=1.85 \mathrm{~K}$, for which the viscosities are almost equal. The viscosity of the normal-fluid component (solid lines) is larger than for the superfluid (dashed lines) for $T=1.65 \mathrm{~K}$ and smaller for $T=2.0 \mathrm{~K}$.

Next, we add the coupling by the mutual friction force, creating a coflow (green and brown lines). The strongly coupled components $\left(V_{0} \Omega_{20}\right.$, brown lines) are well correlated at all scales and move almost as one fluid. The corresponding 
(a)

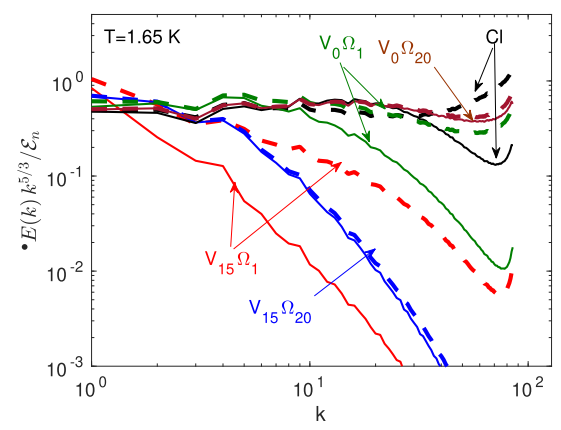

(d)

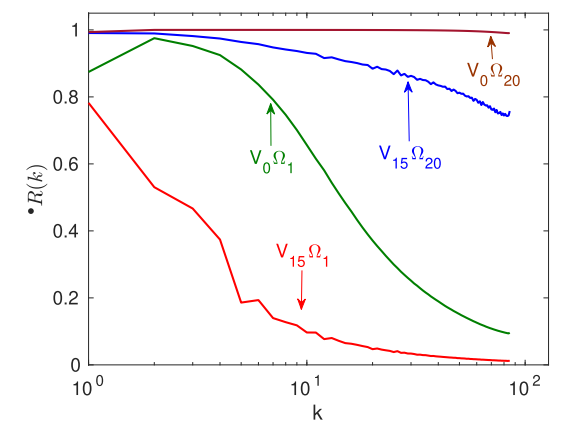

(b)

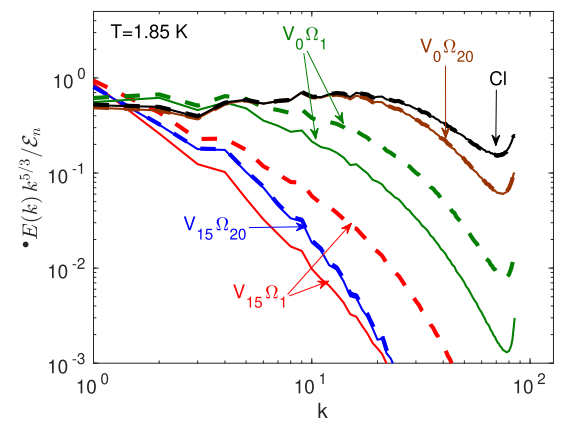

(e)

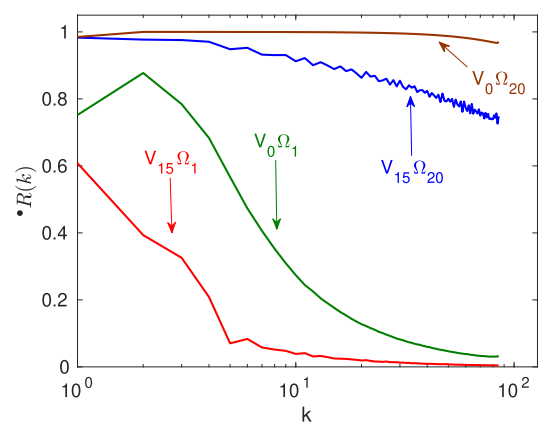

(c)

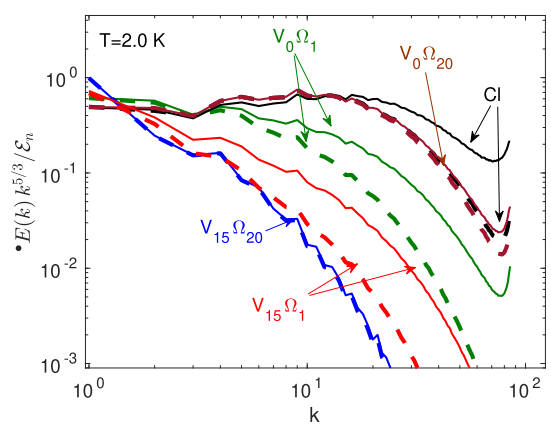

(f)

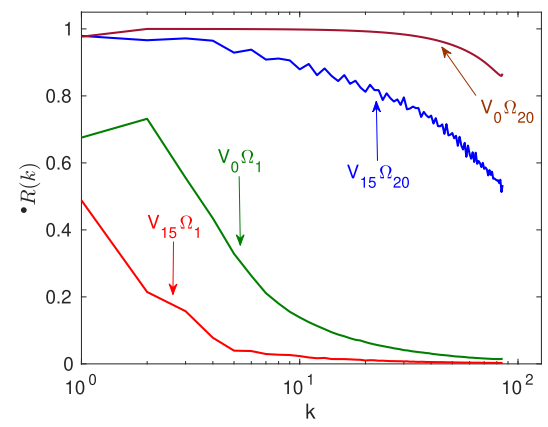

FIG. 1. The spherically averaged energy spectra and the cross-correlation functions for the counterflow and coflow at different temperatures. (a), (b), and (c) The K41-compensated energy spectra ${ }^{\bullet} E_{j}(k)$ for the normal-fluid (solid lines) and superfluid components (dashed lines). (d), (e), and (f) The normalized cross-correlation functions $\bullet R(k)$. The four sets of lines in each panel correspond to $V_{0} \Omega_{1}$, green lines; $V_{0} \Omega_{20}$, brown lines; $V_{15} \Omega_{1}$, red lines; $V_{15} \Omega_{20}$, blue lines. The black lines, labeled "Cl" in (a), (b), and (c) correspond to the spectra of classical turbulence.

spectra slightly differ only at the viscous scales. Note the additional dissipation due to mutual friction, leading to further suppression of the spectra compared to the uncoupled case, for $T=1.85$ and $2.0 \mathrm{~K}$. At the lower temperature $T=1.65 \mathrm{~K}$ the energy exchange between components leads to stronger dissipation in the superfluid component and weaker dissipation in the normal-fluid component. For weaker coupling $\left(V_{0} \Omega_{1}\right.$, green lines), the situation is completely different. The components are almost uncorrelated, especially at large $k$. The coupling between them is translated into very efficient dissipation by mutual friction, leading to spectra that are suppressed almost at all scales, especially at high temperature (see Ref. [20] for details). Note that the slight rise of the classical and coflow spectra at the largest wave numbers is a result of the numerical procedure and does not reflect any underlying physical process.

In the presence of the counterflow velocity [17], the two components are swept in opposite directions by the corresponding mean velocities. This leads to further decorrelation of the component's turbulent velocities, especially at small scales, for which the overlapping time is very short [cf. lines for $V_{15} \Omega_{1}$ and $V_{15} \Omega_{20}$ in Figs. 1(d)-1(f)]. Even for the strong coupling $V_{15} \Omega_{20}$, blue lines, the velocities become progressively less correlated for all temperatures. The dissipation by mutual friction is very strong in this case, with both $\Omega$ and the velocity difference being large, leading to very strongly suppressed spectra, with ${ }^{\bullet} E_{\mathrm{nn}}(k) \approx{ }^{\bullet} E_{\mathrm{ss}}(k)$. At $T=1.65 \mathrm{~K}$ there is still some interval of scales with $k \gtrsim k_{0}$, for which the spectra are close to K41 scaling. The crossover scale agrees well with $k_{\times} \approx 7$ for this case (see Table I, column 14). For higher temperatures this crossover scale becomes smaller and the classical-like behavior is not resolved. At weak coupling $\left(V_{15} \Omega_{1}\right.$, red lines), the velocities are essentially uncorrelated and the spectra of the two components differ and are very strongly suppressed, especially at high $T$.

\section{Angular dependence of 2D-energy spectra}

The behavior of the spherically averaged energy spectra agrees well with the predictions of the theory[18], based on the assumption of spectral isotropy. To explore the angular dependence of the energy spectra and the correlations $\widetilde{E}_{i j}(k, \theta)$ we plot in Figs. 2(a)-2(c) the spectra $\tilde{E}_{j}(k, \theta)$, normalized by the corresponding ${ }^{\bullet} E_{j}(k)$ and in Figs. 2(d)-2(f) the corresponding normalized cross-correlations $\tilde{R}(k, \theta)$. Given the discrete nature of the $\boldsymbol{k}$ space in DNS, we further average them over three bands of wave numbers. We do not account for the largest scales $k \approx k_{0}$ which are influenced by the forcing and average the spectra and the cross-correlations over the $k$ ranges $10 \leqslant k<20,20 \leqslant k<60$, and $60 \leqslant k \leqslant$ 80. The corresponding lines are labeled as $k_{10}, k_{20}$, and $k_{60}$, respectively. Here we consider only strong coupling regime and plot the spectra and the cross-correlations for the coflow $\left(V_{0} \Omega_{20}\right)$ and for the counterflow $\left(V_{15} \Omega_{20}\right)$.

The first observation is that the spectra and the crosscorrelation for the coflow are isotropic for all the conditions. 
(a)

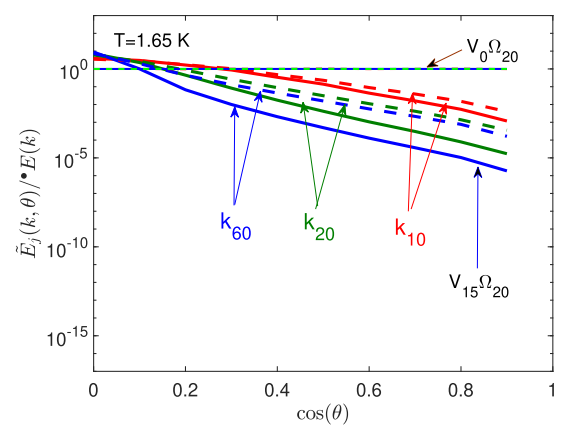

(d)

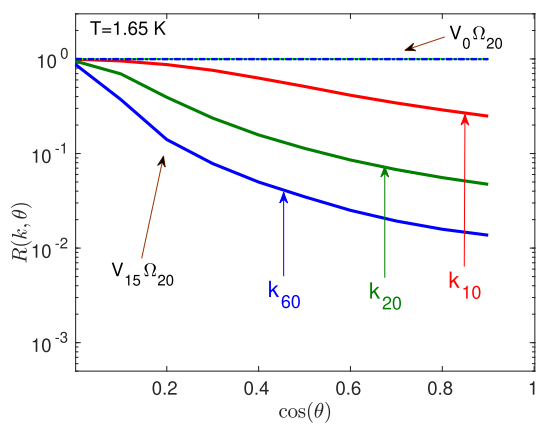

(b)

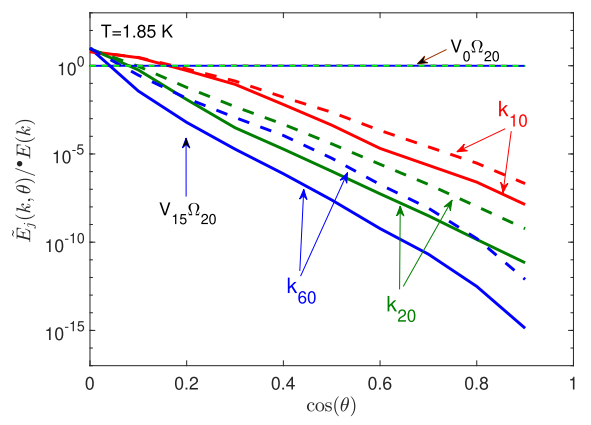

(e)

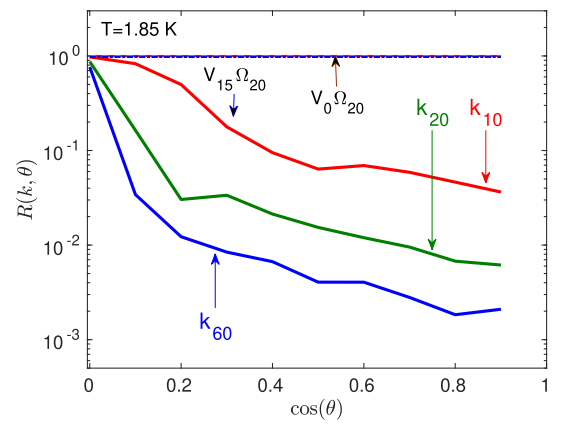

(c)

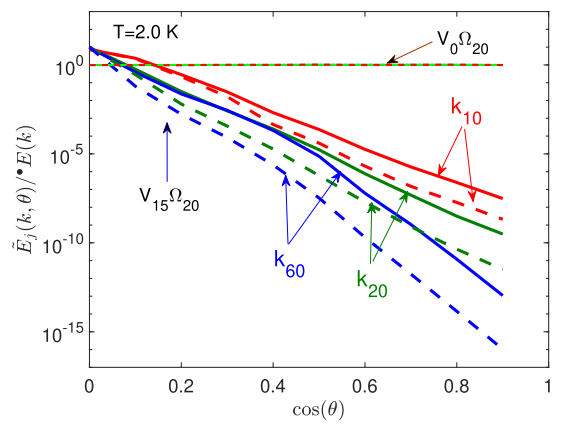

(f)

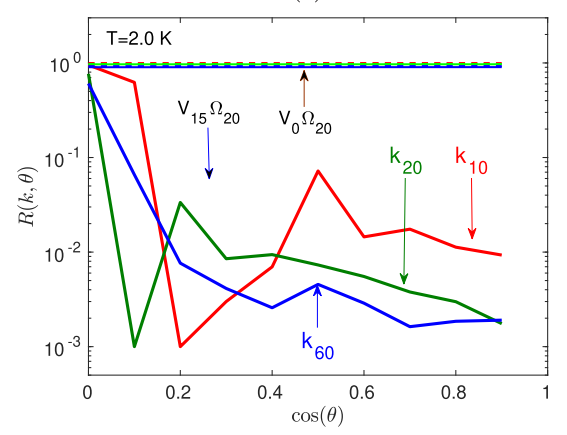

FIG. 2. The angular dependencies of the energy spectra $\tilde{E}_{j}(k, \theta) /{ }^{\bullet} E_{j}(k)$ and the cross-correlations $R(k, \theta)$, averaged in three wave-number bands, for various flow condition. (a), (b), and (c) The 2D-energy spectra $\tilde{E}_{j}(k, \theta)$. The spectra of the normal fluid are shown by solid lines and of the superfluid by dashed lines. (d)-(f) The cross-correlations $R(k, \theta)$. The spectra and the cross-correlation for the coflow are shown by thin horizontal lines and marked $V_{0} \Omega_{20}$, and for the counterflow by thick lines and marked $V_{15} \Omega_{20}$. In all panels, red lines correspond to the averaging over wave-number range $10 \leqslant k<20$ (labeled as $k_{10}$ ), green lines correspond to averaging over $20 \leqslant k<60$ (labeled as $k_{20}$ ), and blue lines correspond to the averaging over $60 \leqslant k \leqslant 80$ (labeled as $k_{60}$ ). Note the log-linear scale.

The angular dependencies of $\tilde{E}_{j}(k, \theta)$ and $\tilde{R}(k, \theta)$ for the counterflow, on the other hand, have a complicated form. Both the spectra and cross-correlation are largest for $\cos \theta \approx 0$ and fall off very quickly with decreasing angle. The spectra decrease exponentially with $\cos \theta$, slower for small $k$ (red lines for $k_{10}$ ) and faster for larger $k$ (green and blue lines for $k_{20}$ and $k_{60}$, respectively). This effect is stronger for the normal-fluid (superfluid) component at low temperatures (high temperature). Most of the energy is contained in the narrow range $\cos \theta<0.1$, near the $\perp$ plane in the $\boldsymbol{k}$ space, orthogonal to $\boldsymbol{U}_{\mathrm{ns}}$.
To better quantify the angular energy distribution, we use the fact that the spectra $\tilde{E}_{j}(k, \theta)$ have piecewise exponential dependence of $\cos \theta$, as is evident from Figs. 2(a)-2(c). We then estimate the $\cos \theta$ range, in which half of the total energy is contained, for different wave-number bands. At $T=1.65 \mathrm{~K}$, for the small wave numbers $k_{10}$ band, this range is indeed $\cos (\theta)<0.1$ for both the normal an superfluid components. With increasing temperature, this range decreases to $\cos \theta<0.05$ for $T=1.85 \mathrm{~K}$ and to $\cos \theta<0.03$ for the normal fluid and $\cos \theta<0.025$ for the superfluid at $T=2.0 \mathrm{~K}$. For the larger $k_{20}$ band these values are 0.04 and 0.06 for the
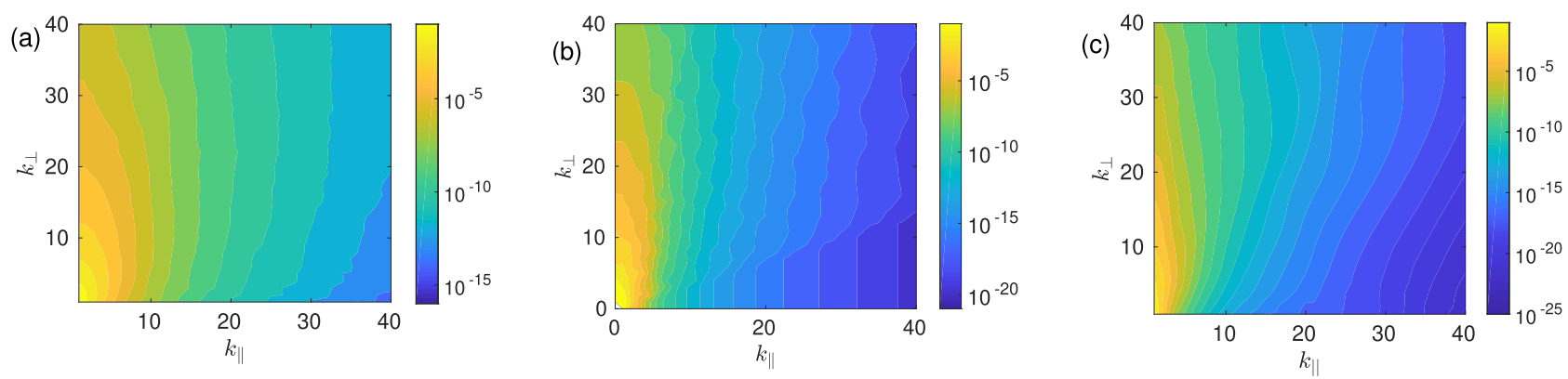

FIG. 3. The superfluid component energy spectrum $E_{\mathrm{s}}\left(k_{\|}, k_{\perp}\right)$ in the counterflow. (a) $T=1.65 \mathrm{~K}$, (b) $T=1.85 \mathrm{~K}$, (c) $T=2.0 \mathrm{~K}$. Note the difference in the magnitudes, shown by the color-bar range. 

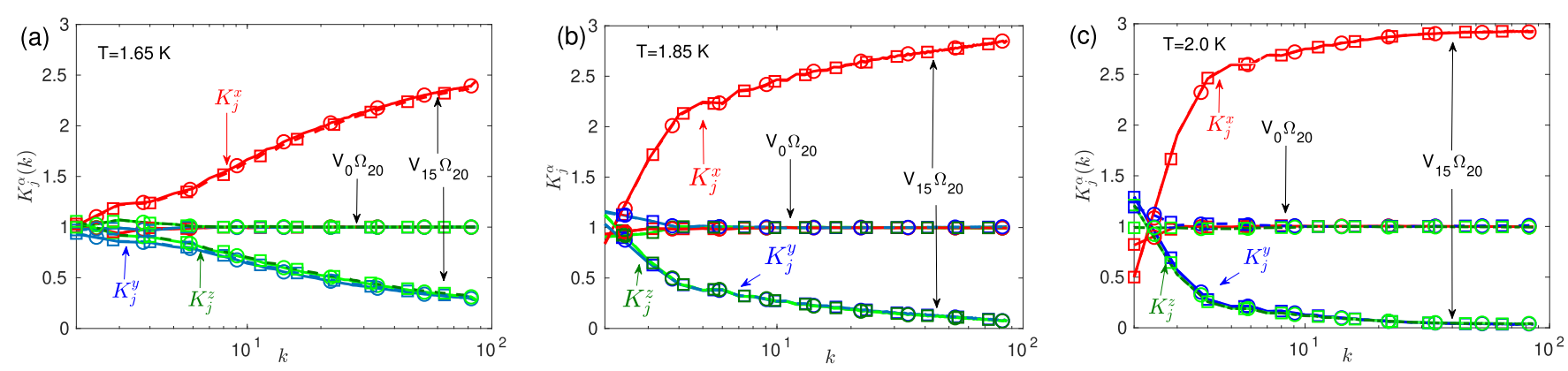

FIG. 4. Tensor decomposition of the $1 \mathrm{D}$ energy spectra $K_{j}^{\alpha}$ for normal-fluid (circles) and superfluid components (squares). The spectra for the coflow are labeled $V_{0} \Omega_{20}$, and for the counterflow $V_{15} \Omega_{20}$. The $K_{j}^{x}, K_{j}^{y}$, and $K_{j}^{z}$ tensor components are annotated and denoted by red, blue, and green lines, respectively.

normal fluid and the superfluid, respectively, at $T=1.65 \mathrm{~K}$. For higher temperatures, as well as for the high wave-number $k_{60}$ band, about half of the total energy is contained in a narrow $\cos \theta<0.02-0.025$ range for both components.

Indeed, the superfluid energy spectrum $E_{\mathrm{ss}}\left(k_{\|}, k_{\perp}\right)$, shown in Fig. 3, is strongly suppressed in the $k_{\|}$direction, while decreasing slowly in the orthogonal direction, especially for $T=2.0 \mathrm{~K}$.

\section{Tensor structure of $1 \mathrm{D}$ energy spectra}

Given such a strong anisotropy of the spectra in the counterflow, it is natural to expect that different components of the turbulent velocity fluctuations are excited to a different extent. In this section we consider the tensor structure of 1D-energy spectra $E_{j j}^{\alpha \alpha}(k)$ for $\alpha=x, y, z$ and clarify which components ( $v_{j}^{x}$ along $\boldsymbol{U}_{\mathrm{ns}}$ or $v_{j}^{y}, v_{j}^{z}$, both orthogonal to $\boldsymbol{U}_{\mathrm{ns}}$ ) are most excited.

In Fig. 4 we plot the components of the spherical spectra for three temperatures as the ratios,

$$
{ }^{\bullet} K_{j}^{\alpha}(k) \equiv 3^{\bullet} E_{j j}^{\alpha \alpha}(k) /{ }^{\bullet} E_{j j}(k)
$$

The factor 3 was introduced to ensure that for the isotropic turbulence $\sum_{\alpha=x, y, z}{ }^{\circ} K_{j}^{\alpha}(k)=1$.

Indeed, for the coflow (the almost horizontal lines, labeled $V_{0} \Omega_{20}$ ) all the velocity components are excited equally, except for the smallest wave numbers. On the other hand, for the

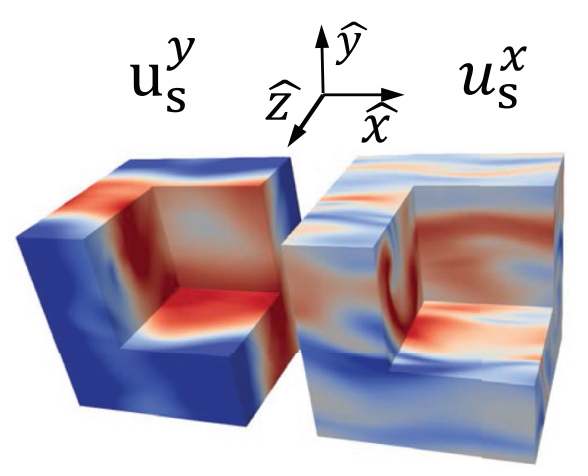

FIG. 5. Superfluid velocity $u_{\mathrm{s}}^{x}$ and $u_{\mathrm{s}}^{y}$ components [50]. $T=$ $1.85 \mathrm{~K}, \Omega=20, V=15$. The $u_{\mathrm{s}}^{z}$ component (not shown) is similar to the $y$ component. The velocity magnitude is color coded, with red denoting positive and blue denoting negative values. counterflow turbulence (lines labeled $V_{15} \Omega_{20}$ ) the contribution of the ${ }^{\bullet} K_{j}^{x}(k)$ component (shown by red lines) is dominant and monotonically increasing with $k$ from the isotropic level ${ }^{-} K_{j}^{x}\left(k_{0}\right) \approx 1$ to the maximal possible level ${ }^{\bullet} K_{j}^{x}(k) \approx 3$. This means that the small-scale counterflow turbulence mainly consists of $v_{j}^{x}(\boldsymbol{k})$ velocity fluctuations. The contribution of $v_{j}^{y}$ and $v_{j}^{z}$ fluctuations for $k \gtrsim 10$ is negligible, especially at $T=2.0 \mathrm{~K}$.

Therefore, the counterflow turbulence represent a special kind of a quasi-2D turbulence, consisting mostly of the turbulent velocity fluctuations with only one streamwise projection $\boldsymbol{u}_{\|}$, which depends on the cross-stream coordinate $\boldsymbol{r}_{\perp}$ : $\boldsymbol{u}_{\|}\left(\boldsymbol{r}_{\perp}, t\right)$. This behavior is essentially different from other known types of quasi-2D turbulence, such as stably stratified flow in the atmosphere [23-25] or rotational turbulence [26-28], in which the leading contribution to the turbulent velocity field comes from the 2D velocity field $\boldsymbol{u}_{\perp}$ that depends on $\boldsymbol{r}_{\perp}: \boldsymbol{u}_{\perp}\left(\boldsymbol{r}_{\perp}, t\right)$. Such a $\boldsymbol{u}_{\|}\left(\boldsymbol{r}_{\perp}, t\right)$ turbulence can be visually presented as narrow jets or thin sheets as illustrated in Fig. 5.

Note the difference with the strong acoustic turbulence. There the velocity field has tangential velocity breaks at the jets' boundaries and the $1 \mathrm{D}$ energy spectrum $E(k) \propto k^{-2}$. The energy spectra in the counterflow decay much faster. It means that the velocity fields at the jets' boundaries are continuous together with some finite number of their derivatives. This is a consequence of the mutual friction that tends to smooth the velocity field.

\section{E. Comparison of $1 D$ energy spectra and reconstruction of $3 \mathrm{D}$ spectra}

The best way to study the anisotropy of hydrodynamic turbulence is to expand the statistical objects in the irreducible representations of the $\mathrm{SO}(3)$ symmetry group (see, e.g., Refs. [25,46-49]). In counterflow turbulence, an attempt to expand $\tilde{E}_{j}(k, \theta)$ into a series with respect to Legendre polynomials,

$$
\tilde{E}_{j}(k, \theta)=\sum_{\ell} \tilde{E}_{j}(k, \ell) P_{\ell}(\cos \theta),
$$

and to study $k$ behavior of $\tilde{E}_{j}(k, \theta)$, turned out to be ineffective. The very strong anisotropy of $\tilde{E}_{j}(k, \theta)$ spectra required too many terms in the expansion (22) for an adequate reproduction of its angular dependence. Therefore, we choose another way to characterize the spectral anisotropy, which is more suitable in our case. We compare the normal-fluid and 
(a)

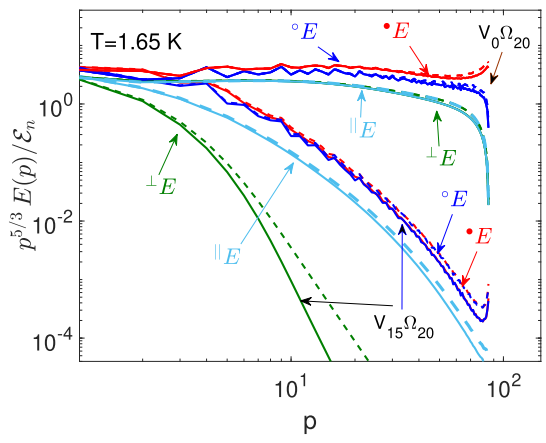

(b)

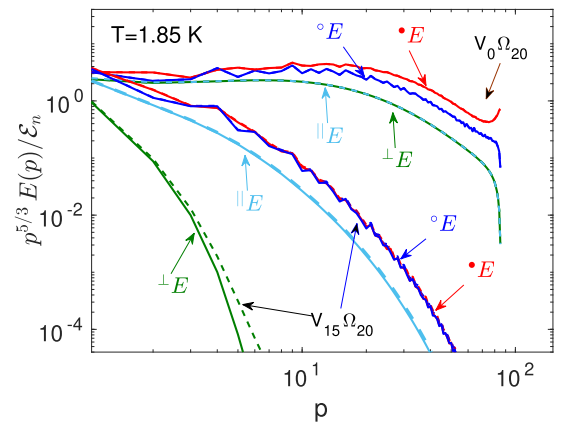

(c)

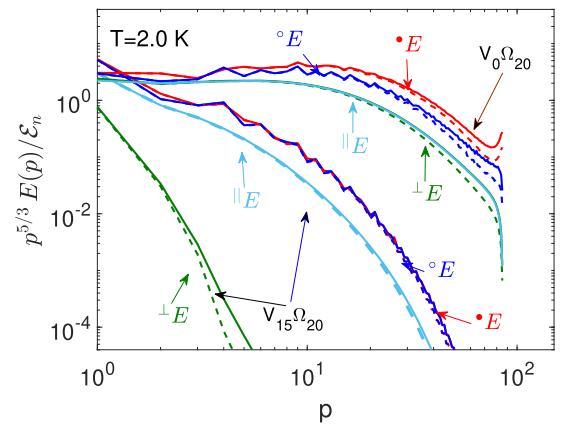

FIG. 6. Various 1D energy spectra of the normal-fluid (solid lines) and the superfluid (dashed) components for the coflow (lines labeled $V_{0} \Omega_{20}$ ) and for the counterflow (lines labeled $V_{15} \Omega_{20}$ ). Different kinds of spectra are annotated in the figure.

superfluid spherical ${ }^{\bullet} E_{j}(k)$, cylinder ${ }^{\circ} E_{j}\left(k_{\perp}\right)$, and $\|$-, $\perp$-planeaveraged energy spectra ${ }^{\|} E_{j}\left(k_{\perp}\right),{ }^{\perp} E_{j}\left(k_{\|}\right)$.

In the case of isotropy, all the four 1D energy spectra are proportional to each other,

$$
{ }^{\bullet} E_{i j}^{\alpha \beta}(p) \propto{ }^{\circ} E_{i j}^{\alpha \beta}(p) \propto{ }^{\perp} E_{i j}^{\alpha \beta}(p) \propto{ }^{\|} E_{i j}^{\alpha \beta}(p),
$$

differing only in numerical prefactors. Here $p$ is the corresponding (dimensional, $[p]=1 / \mathrm{cm}$ ) wave number: $p=$ $k, k_{\perp}, k_{\|}$, or $k_{y}$. By estimating contributions to the integrals in Eqs. (7a), (7c), and (7e) in the case of strong anisotropy (i.e., coming from a narrow range with $k_{\|} \ll k_{\perp}$ ), one may show that the spectra are related as ${ }^{\bullet} E_{j}(p) \approx{ }^{\circ} E_{j}(p) \approx C^{\|} E_{j}(p)$, where $C$ is a numerical prefactor. This fact may explain the good agreement between the experimental spectra ${ }^{\|} E_{\mathrm{n}}\left(k_{\perp}\right)$, obtained in Ref. [13] and the prediction of the theory [18] for $E_{j}(k)$. The integral in Eq. (7d) is different and the spectrum ${ }^{\perp} E_{j}\left(k_{\|}\right)$is expected to be confined to small $k_{\|}$range.

These spectra, normalized by the energy density $\mathcal{E}_{\mathrm{n}}$ and compensated by the $\mathrm{K} 41$ factor $p^{5 / 3}$, are shown in Fig. 6 . The coflow spectra, appearing as almost horizontal lines, labeled $V_{0} \Omega_{20}$, indeed differ by less than an order of magnitude for all $T$. The relation between various spectra for the counterflow is consistent with the above estimate, further confirming the strong spectral anisotropy. The degree to which the ${ }^{\perp} E_{j}(p)$ spectra, shown by green lines, are suppressed at different temperatures, agrees with the angular dependence of $\tilde{E}_{j}(k, \theta)$, Figs. 2(a)-2(c). While at $T=1.65 \mathrm{~K}$ the spectrum for the $k_{10}$

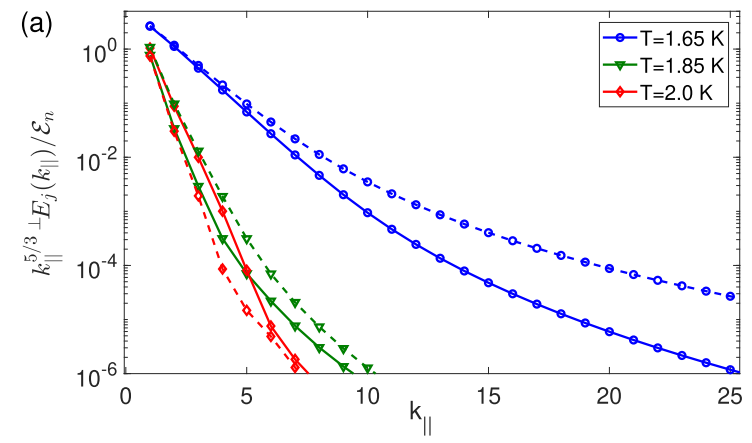

range at $\cos \theta \approx 1$ is smaller by three orders of magnitude in the direction of the counterflow compared to the orthogonal plane, at $T=2.0 \mathrm{~K}$ this difference is almost 10 orders of magnitude. Accordingly, the ${ }^{\perp} E_{j}(p)$ spectrum at $T=2.0 \mathrm{~K}$ is confined to less than a decade in $p$. To better quantify the steepness of the spectra we list in Table II the values of the ratios $E_{j}(10) / E_{j}(1)$ for ${ }^{\perp} E_{j}(p)$ and ${ }^{\|} E_{j}(p)$.

The analysis of the $(k, \theta)$ dependence of the $\tilde{E}_{j}(k, \theta)$ energy spectra in Sec. IIIC showed that the overwhelming part of the total turbulent energy is concentrated in the range of small $\cos \theta$, and consequently small $k_{\|}$, say for $k_{\|} \lesssim 10$. For a semiqualitative analysis of the $2 \mathrm{D}$ spectra $E_{j}\left(k_{\|}, k_{\perp}\right)$ and $\tilde{E}_{j}(k, \theta)$ in this range of $k_{\|}$, we assume a factorization,

$$
E\left(k_{\|}, k_{\perp}\right) \simeq f_{1}\left(k_{\|}\right) f_{2}\left(k_{\perp}\right) .
$$

If so, using Eqs. (7c) and (7d), we can reconstruct the 2Denergy spectra as follows:

$$
E_{j}\left(k_{\|}, k_{\perp}\right) \simeq \frac{{ }^{\circ} E_{j}\left(k_{\perp}\right)^{\perp} E_{j}\left(k_{\|}\right)}{E_{j}},
$$

where $E_{j}$ is the energy density in the system given by Eq. (8).

Furthermore, using Eq. (6), we can also reconstruct the 2D spectra $\tilde{E}(k, \theta)$ from $E\left(k_{\|}, k_{\perp}\right)$ :

$$
\tilde{E}_{j}(k, \theta) \simeq \frac{{ }^{\circ} E_{j}(k \sin \theta)^{\perp} E_{j}(k \cos \theta)}{E_{j} \sin \theta} .
$$

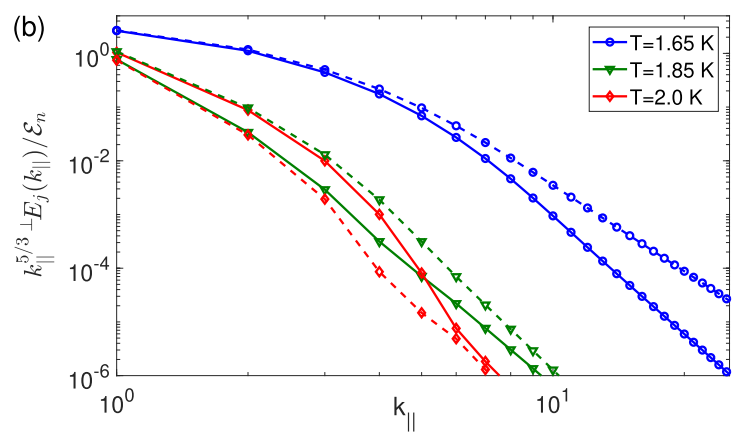

FIG. 7. The $\perp$-plane energy spectra ${ }^{\perp} E_{j}\left(k_{\|}\right)$of the normal-fluid (solid lines) and the superfluid (dashed) components for counterflow $V_{15} \Omega_{20}$. The spectra are shown in a log-linear scale in (a) and in the log-log scale in (b). Different temperatures are annotated in the figure. Note linear behavior at small $k_{\|}$in (a) and at large $k_{\|}$in (b). 
TABLE II. The steepness of the energy spectra ${ }^{\perp} E_{j}(k)$ and ${ }^{\|} E_{j}(k)$ in the counterflow, characterized by the ratios ${ }^{\perp} E_{j}(10) /{ }^{\perp} E_{j}(1)$ and ${ }^{\|} E_{j}(10) /{ }^{\|} E_{j}(1)$.

\begin{tabular}{lccc}
\hline \hline & $T=1.65 \mathrm{~K}$ & $T=1.85 \mathrm{~K}$ & $T=2.0 \mathrm{~K}$ \\
\hline${ }^{\|} E_{\mathrm{n}, \mathrm{s}}(10) /{ }^{\|} E_{\mathrm{n}, \mathrm{s}}(1)$ & $10^{-3}$ & $2.0 \times 10^{-4}$ & $2.5 \times 10^{-4}$ \\
${ }^{\perp} E_{\mathrm{n}}(10) /{ }^{\perp} E_{\mathrm{n}}(1)$ & $6.3 \times 10^{-6}$ & $2.0 \times 10^{-8}$ & $1.6 \times 10^{-8}$ \\
${ }^{\perp} E_{\mathrm{s}}(10) /{ }^{\perp} E_{\mathrm{s}}(1)$ & $2.5 \times 10^{-5}$ & $3.2 \times 10^{-8}$ & $1.6 \times 10^{-9}$ \\
\hline \hline
\end{tabular}

In the range of small $\cos \theta$, where most of the turbulent energy is concentrated, Eq. (24c) can be simplified as follows: $\tilde{E}_{j}(k, \theta) \simeq{ }^{\circ} E_{j}(k){ }^{\perp} E_{j}(k \cos \theta) / E_{j}$. The $\theta$ dependence of $\tilde{E}_{j}(k, \theta)$ is therefore determined by ${ }^{\perp} E_{j}(k \cos \theta)$, i.e., $\cos \theta$ appears in the combination $k \cos \theta=k_{\|}$. This observation fully agrees with our theoretical prediction, that $\cos \theta$ appears in the theory only via Eq. (16c) in the dimensionless factor $k U_{\mathrm{ns}} \cos \theta / \Omega_{\mathrm{ns}}$. We consider this agreement as an argument in a favor of the factorization assumption (24a) for small $\cos \theta$.

To take a closer look at ${ }^{\perp} E_{j}\left(k_{\|}\right)$, we plot in Fig. 7 these spectra for different temperatures. To expose the functional dependence of the spectra, we use different scales in two panels: in Fig. 7(a) the scale is log-linear, while in Fig. 7(b) the spectra are plotted in the log-log scale. At all temperatures the small- $k_{\|}$behavior is exponential, while at larger $k_{\|}$the spectra are consistent with the power-law behavior. Using this information, we propose the following form for the small- $k_{\|}$ spectra:

$$
{ }^{\perp} E_{j}\left(k_{\|}\right) \simeq \frac{k_{\|} \mathcal{E}_{j}}{k_{*}} \exp \left[-\frac{k_{\|}}{k_{*}}\right] .
$$

It is tempting to relate the characteristic $k_{*}$ to the crossover scale $k_{\times}: k_{*} \propto \Omega_{\mathrm{ns}} / U_{\mathrm{ns}}=k_{\times}$. Indeed, $k_{*}$ estimated from Fig. 7(a) and $k_{\times}$(Table I, column 14) have similar temperature trends. This gives additional support for factorization (24a) and for qualitative theoretical discussion of the problem in Sec. II C.

The observed steep power-law behavior of ${ }^{\perp} E_{j}\left(k_{\|}\right)$for larger $k_{\|} \gtrsim 10$, Fig. 7(b) with an apparent exponent $m \approx-7$ may indicate a nonlocal energy transfer between largest and smallest scales, similar to the supercritical spectra in the superfluid ${ }^{3} \mathrm{He}$.

\section{F. The structure functions}

The energy spectra ${ }^{\|} E_{j}\left(k_{\perp}\right),{ }^{\perp} E_{j}\left(k_{\|}\right)$may be translated into the corresponding structure functions, according to Eqs. (10) and (13). In Fig. 8 we show the structure functions (9b) $S_{n}^{\|}(R) \equiv S_{\mathrm{n}}^{x x}(R)$ with the velocity differences taken in the direction of the counterflow $R_{\|} \equiv R_{x}$ and in the plane $R_{\perp}$ orthogonal to it. The structure functions (9b) for the coflow, shown in Figs. 8(a)-8(c) as red and orange lines are similar to classical turbulence; at large scales they follow approximately $R^{2 / 3}$ scaling, gradually crossing over towards viscous $R^{2}$ behavior. The transition is very broad here, but the two apparent scaling ranges are evident. The cross-over scale increases with temperature. The structure functions, calculated along and across the counterflow direction, are similar, slightly differing mostly in the magnitude at all scales. The main difference from the uncoupled case (not shown) is the lower magnitude at all scales, reflecting the presence of additional energy dissipation by mutual friction. In the counterflow, the situation is different. Over most of the available range of scales, the structure functions, shown as dark and light blue lines in Fig. 8, appear to have an apparent scaling behavior close to $R^{2}$, especially $S_{\mathrm{n}}^{\|}\left(R_{||}\right)$. The actual behavior depends on the flow conditions, in agreement with the results of Ref. [12]. The magnitudes of the structure functions are much lower than for the coflow. At the lower temperature $T=1.65 \mathrm{~K}, S_{\mathrm{n}}^{\|}(R \perp)$ has an overlap with the corresponding structure function in the coflow a large scale, which disappears with increasing temperature. The two types of the structure functions in the counterflow have significant difference in magnitude, with $S_{\mathrm{n}}^{\|}\left(R_{\|}\right)$being strongly suppressed. As it was suggested in Sec. II B 2, these structure functions do not quantitatively reflect the corresponding energy spectra, however, the qualitative difference should be observable experimentally.

The influence of the coupling strength on the behavior of the structure functions is illustrated in Fig. 9 for $T=1.65 \mathrm{~K}$. Here, in addition to the weak coupling $\Omega=1$ and the strong coupling $\Omega=20$ we consider also an intermediate coupling strength $\Omega=3.4$. The structure functions for the classical turbulence are included for comparison. The general form is similar for all values of $\Omega$, with all the structure functions in the counterflow being strongly suppressed compared to classical turbulence, especially $S_{j}^{\|}\left(R_{\|}\right)$. Note that at this temperature (a)

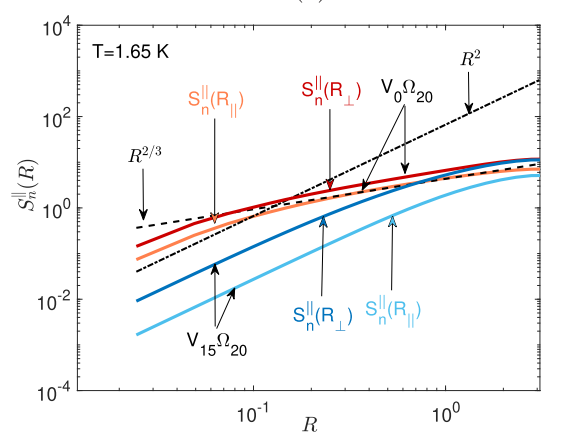

(b)

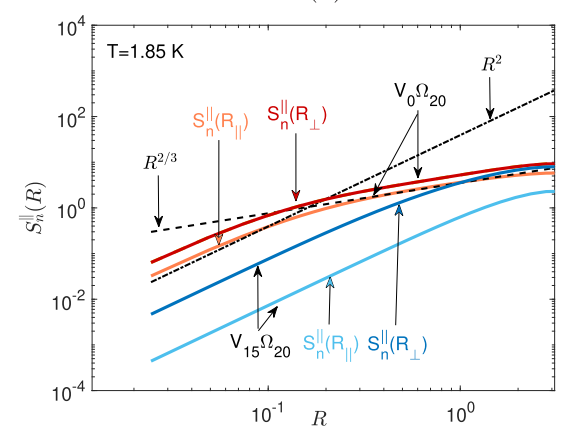

(c)

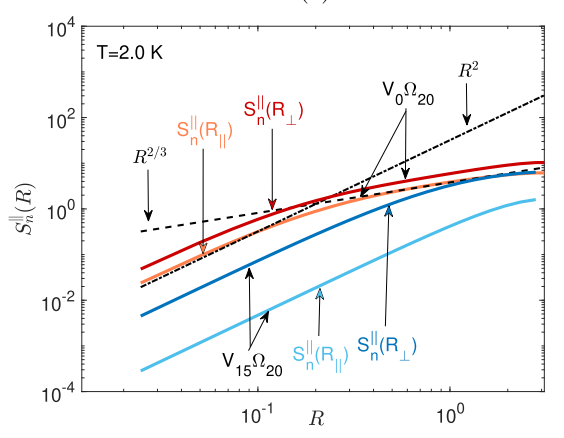

FIG. 8. The velocity structure functions of the normal-fluid component $S_{\mathrm{n}}^{\|}(R)(9 \mathrm{~b})$. The lines for the coflow are marked $V_{0} \Omega_{20}$, and for the counterflow $V_{15} \Omega_{20}$. Various structure functions are marked in the figures. The dashed lines labeled $R^{2 / 3}$ and the dot-dashed lines marked $R^{2}$ serve to guide the eye only. 

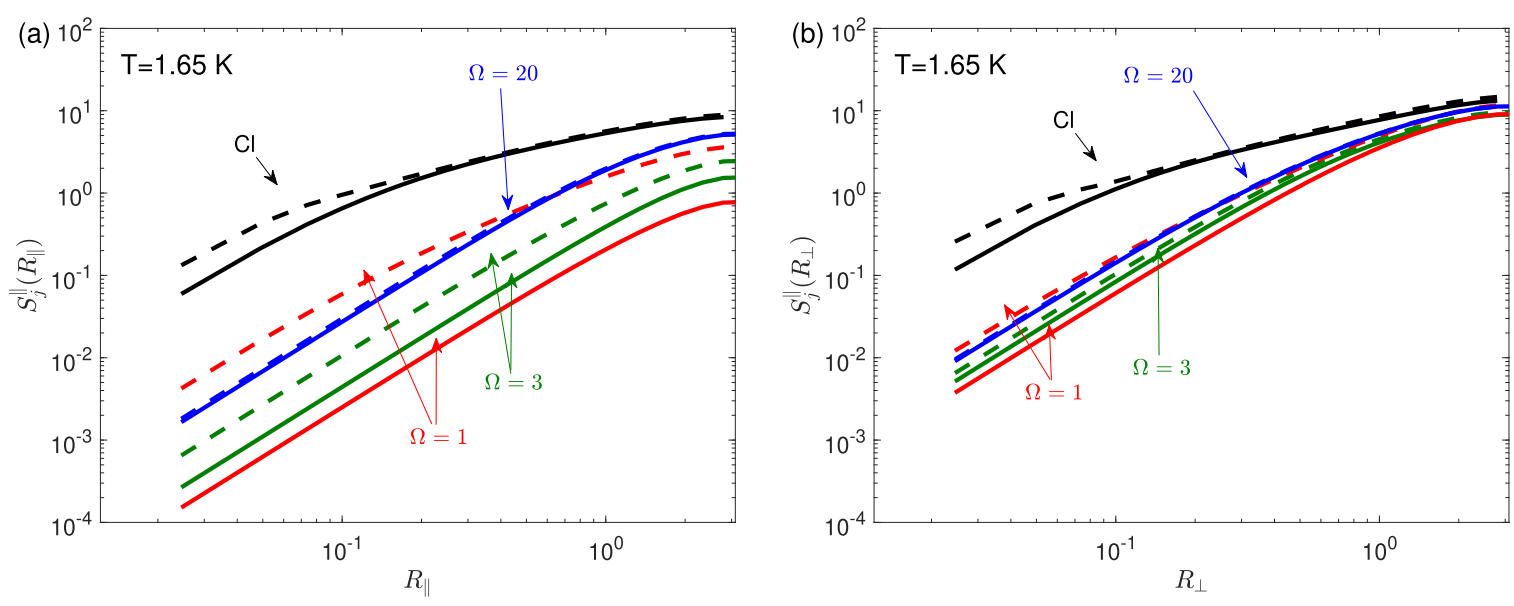

FIG. 9. The structure functions $S_{j}^{\|}\left(R_{\|}\right)$(a) and $S_{j}^{\|}\left(R_{\perp}\right)$ (b) at $T=1.65 \mathrm{~K}$. The lines for the counterflow with $V=15$ and different values of $\Omega$ are color coded: $\Omega=1$ (red lines), $\Omega=3$ (green lines), $\Omega=20$ (blue lines). The structure functions for the normal-fluid component are shown by solid lines and for the superfluid component by dashed lines. Structure functions for the classical turbulence $(V=0, \Omega=0)$ are shown by black lines and labeled $\mathrm{Cl}$.

the structure functions of the normal fluid are more suppressed for weaker coupling, in accordance with the energy spectra in Fig. 1(a). For the transverse $S_{j}^{\|}\left(R_{\perp}\right)$ the difference between the two fluid components is relatively small and the influence of the coupling strength is weak. This is consistent with the 2D-energy spectra, shown in Fig. 3: The energy spectra in the transverse direction $k_{\perp}$ are weakly influenced by the mutual friction.

Additional information may be obtained from analysis of the flatness $F_{j}^{\|}(R)=P_{j}^{\|}(R) /\left[S_{j}^{\|}(R)\right]^{2}$, where $P_{j}^{\|}(R)=$ $\left.\left\langle\left(\delta_{\boldsymbol{R}} u_{j}^{x}\right)^{4}\right)\right\rangle$ is the fourth-order structure function. In Fig. 10 we compare $F_{\mathrm{n}}^{\|}\left(R_{\perp}\right)$ and $F_{\mathrm{n}}^{\|}\left(R_{\|}\right)$with the flatness in the

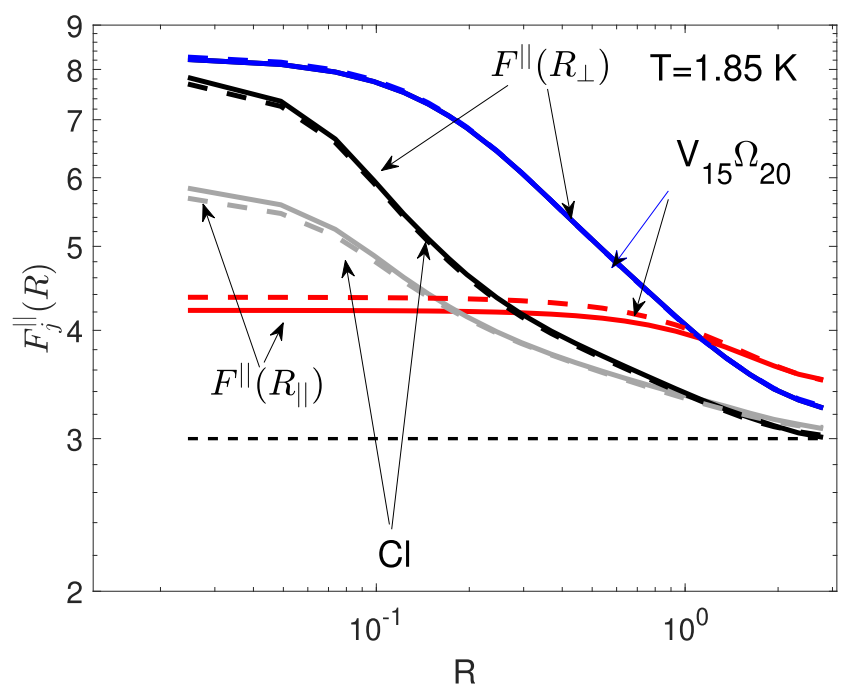

FIG. 10. The flatness $F_{j}^{\|}\left(R_{\|}\right)$(red lines) and $F_{j}^{\|}\left(R_{\perp}\right)$ (blue lines) $T=1.85 \mathrm{~K}$ in the counterflow $\Omega_{\mathrm{s}}=20, V=15$. The flatness for the normal-fluid component are shown by solid lines and for the superfluid component by dashed lines. The corresponding flatnesses for the classical turbulence $(V=0, \Omega=0)$ are shown by black and gray lines and labeled $\mathrm{Cl}$. classical turbulence. In the transverse direction, $F_{\mathrm{n}}^{\|}\left(R_{\perp}\right)$ in the counterflow is growing towards small scales faster than in the classical turbulence at large scales. This indicates a moderate enhancement of intermittency at intermediate scales in this direction, in agreement with experimental results of Ref. [13]. In the longitudinal direction, similar to the structure functions, the flatness $F_{\mathrm{n}}^{\|}\left(R_{\|}\right)$is almost constant in the counterflow, leading to a much stronger discrepancy between the transverse and the longitudinal components than in the classical turbulence. This constant value reflects the behavior of the structure functions $S_{\mathrm{n}}^{\|}\left(R_{\|}\right) \propto R_{\|}^{2}$ and $P_{\mathrm{n}}^{\|}\left(R_{\|}\right) \propto R_{\|}^{4}$ over a wide range of scales that is a consequence of the energy spectra ${ }^{\perp} E_{j}\left(k_{\|}\right)$that fall off faster than $k^{-3}$.

The second difference structure functions $\tilde{S}_{j}^{\|}(R)(12 \mathrm{~b})$ are expected to better reflect the underlying spectra, at least for ${ }^{\|} E\left(k_{\perp}\right)$, since they have wider windows of locality up to $k^{-5}$. The steeper ${ }^{\perp} E\left(k_{\|}\right)$result in $S^{\|}\left(R_{\|}\right)$behaving as $R^{4}$ in most of the available range of scales. In principle, these exponents can fall within the windows of locality for the structure functions of the third difference (up to $x \leqslant 7$ ) and of the fourth difference (up to $x \leqslant 9.0$ ). We do not discuss these objects due to the increasing difficulties in their measurements.

Having in mind possible experiments, we compare in Fig. 11 two types of the structure functions for the normal fluid in the counterflow. To allow a meaningful comparison we plot them normalized by the values at the largest $R=R_{\max }$ and compensated by the corresponding viscous scaling,

$$
\begin{gathered}
{ }^{\dagger} S_{j}^{\|}(R)=R^{2} S_{j}^{\|}(R) / S_{j}^{\|}\left(R_{\max }\right), \\
{ }^{\dagger} \tilde{S}_{j}^{\|}(R)=R^{4} \tilde{S}_{j}^{\|}(R) / \tilde{S}_{j}^{\|}\left(R_{\max }\right) .
\end{gathered}
$$

Indeed, the transition to the viscous behavior (the horizontal lines at small scales) occurs at smaller $R$ for $\tilde{S}_{j}^{\|}(R)$ (marked by triangles and diamonds) than for $S_{j}^{\|}(R)$ (marked by squares and circles). As expected, the range of the conditiondependent apparent scaling at large scales also increases. In addition, the difference in the amplitudes of the structure functions in the longitudinal and transverse directions is much 
(a)

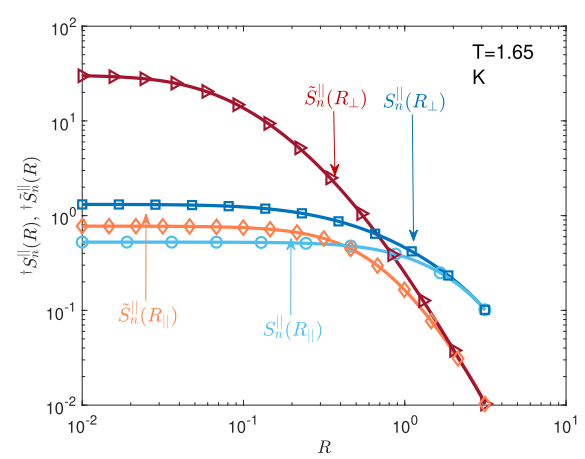

(b)

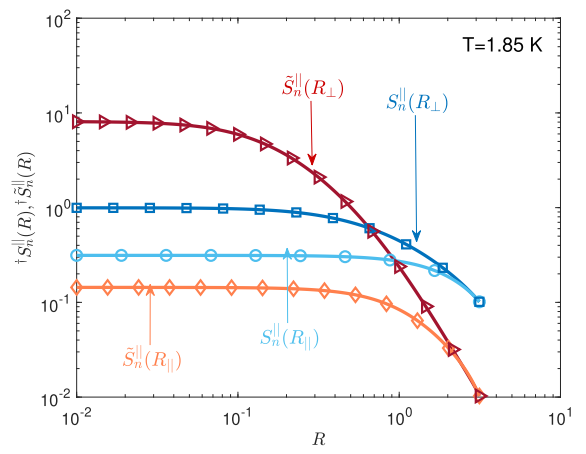

(c)

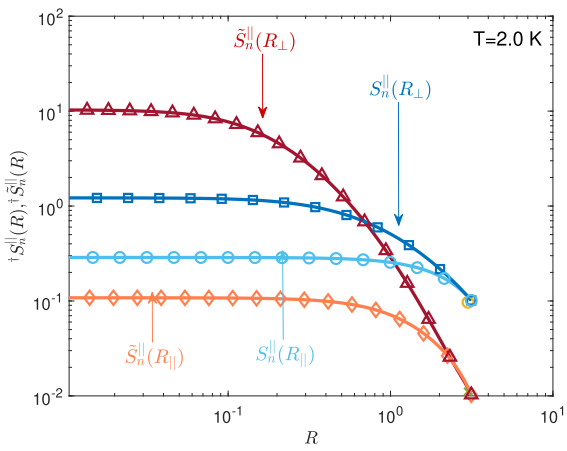

FIG. 11. Comparison of the normalized compensated velocity structure functions ${ }^{\dagger} S_{\mathrm{n}}^{\|}\left(R_{\perp}\right)(\square)$ and ${ }^{\dagger} S_{\mathrm{n}}^{\|}\left(R_{\|}\right)(\bigcirc)$ with ${ }^{\dagger} \tilde{S}_{\mathrm{n}}^{\|}\left(R_{\perp}\right)(\triangleright)$ and ${ }^{\dagger} \tilde{S}_{\mathrm{n}}^{\|}\left(R_{\|}\right)(\diamond)$ for the normal-fluid component in the counterflow.

larger for $\tilde{S}^{\|}(R)$, hopefully allowing more accurate detection of the anisotropy.

\section{CONCLUSIONS}

Both the theoretical considerations and the results of the numerical simulations presented indicate strong anisotropy in the energy distribution in counterflow turbulence. This is basically due to an angular dependence of the energy dissipation caused by the mutual friction force. It tends to suppress the velocity fluctuations elongated across the direction of the counterflow velocity. At the same time, most of the flow energy is confined to a narrow wave-number plane, orthogonal to this direction, leading to a flow which is smooth along the counterflow direction and turbulent across it. Unlike rotational and atmospheric turbulence with stable stratification, in counterflow turbulence the streamwise velocity component plays the dominant role. This effect is progressively stronger at smaller scales and at higher temperatures. At low temperatures, the milder gradual increase of the small-scale anisotropy is due to the smaller fraction of normal fluid and consequently weaker decorrelation. The structure functions of this anisotropic, nonscale invariant turbulent flow, do not allow one to extract the quantitative information about the energy distribution over scales, but are expected to reveal strong differences between the directions along and orthogonal to the counterflow velocity.

\section{ACKNOWLEDGMENTS}

L.B. thanks Michele Buzzicotti for the data analysis and visualization. G.S. thanks AtMath collaboration at University of Helsinki. A.P. and V.L. acknowledge a useful discussion with L. Skrbek. We acknowledge funding from the Prace project "Superfluid Turbulence under counterflows" Pra12 3088 .
[1] R. J. Donnelly, Quantized Vortices in Hellium II (Cambridge University Press, Cambridge, 1991).

[2] Quantized Vortex Dynamics and Superfluid Turbulence, edited by C. F. Barenghi, R. J. Donnelly, and W. F. Vinen, Lecture Notes in Physics 571 (Springer-Verlag, Berlin, 2001).

[3] W. F. Vinen and J. J. Niemela, Quantum turbulence, J. Low Temp. Phys. 128, 167 (2002).

[4] R. P. Feynman, Application of quantum mechanics to liquid helium, Prog. Low Temp. Phys. 1, 17 (1955).

[5] H. E. Hall and W. F. Vinen, The rotation of liquid helium II. I. Experiments on the propagation of second sound in uniformly rotating helium II, Proc. R. Soc. A 238, 204 (1956).

[6] I. L. Bekarevich, and I. M. Khalatnikov, Phenomenological derivation of the equations of vortex motion in He II, Sov. Phys. JETP 13, 643 (1961).

[7] W. F. Vinen, Mutual friction in a heat current in liquid helium II I. Experiments on steady heat currents, Proc. R. Soc. 240, 114 (1957); Mutual friction in a heat current in liquid helium II. II. Experiments on transient effects, 240, 128 (1957); Mutual friction in a heat current in liquid helium II III. Theory of the mutual friction, 242, 493 (1957); Mutual friction in a heat current in liquid helium. II. IV. Critical heat currents in wide channels, 243, 400 (1958).

[8] R. N. Hills and P. H. Roberts, Superfluid mechanics for a high density of vortex lines, Arch. Ration. Mech. Anal. 66, 43 (1977).

[9] K. W. Schwarz, Three-dimensional vortex dynamics in superfluid ${ }^{4} \mathrm{He}$ : Homogeneous superfluid turbulence, Phys. Rev. B 38, 2398 (1988).

[10] L. Skrbek and K. R. Sreenivasan, in Ten Chapters in Turbulence, edited by P. A. Davidson, Y. Kaneda, and K. R. Sreenivasan (Cambridge University Press, Cambridge, 2013), pp. 405-437.

[11] A. Marakov, J. Gao, W. Guo, S. W. Van Sciver, G. G. Ihas, D. N. McKinsey, and W. F. Vinen, Visualization of the normal-fluid turbulence in counterflowing superfluid ${ }^{4} \mathrm{He}$, Phys. Rev. B 91, 094503 (2015).

[12] J. Gao, E. Varga, W. Guo, and W. F. Vinen, Energy spectrum of thermal counterflow turbulence in superfluid Helium-4, Phys. Rev. B 96, 094511 (2017).

[13] S. Bao, W. Guo, V. S. L'vov, and A. Pomyalov, Statistics of turbulence and intermittency enhancement in superfluid ${ }^{4} \mathrm{He}$ counterflow, Phys. Rev. B 98, 174509 (2018). 
[14] M. La Mantia and L. Skrbek, Quantum, or classical turbulence? Europhys. Lett. 105, 46002 (2014).

[15] M. La Mantia, P. Švančara, D. Duda, and L. Skrbek, Small-scale universality of particle dynamics in quantum turbulence, Phys. Rev. B 94, 184512 (2016).

[16] M. La Mantia, Particle dynamics in wall-bounded thermal counterflow of superfluid helium, Phys. Fluids 29, 065102 (2017).

[17] D. Khomenko, V. S. L'vov, A. Pomyalov, and I. Procaccia, Counterflow induced decoupling in superfluid turbulence, Phys. Rev. B 93, 014516 (2016).

[18] V. S. L'vov and A. Pomyalov, A theory of counterflow velocity dependence of superfluid ${ }^{4} \mathrm{He}$ turbulence statistics, Phys. Rev. B 97, 214513 (2018).

[19] L. Biferale, D. Khomenko, V. L'vov, A. Pomyalov, I. Procaccia, and G. Sahoo, Superfluid Helium in Three-Dimensional Counterflow Differs Strongly from Classical Flows: Anisotropy on Small Scales, Phys. Rev. Lett. 122, 144501 (2019).

[20] L. Biferale, D. Khomenko, V. L'vov, A. Pomyalov, I. Procaccia, and G. Sahoo, Turbulent statistics and intermittency enhancement in coflowing superfluid ${ }^{4} \mathrm{He}$, Phys. Rev. Fluids 3, 024605 (2018)

[21] L. Boué, V. S. L’vov, A. Pomyalov, and I. Procaccia, Energy spectra of superfluid turbulence in ${ }^{3} \mathrm{He}$, Phys. Rev. B 85, 104502 (2012).

[22] L. Biferale, D. Khomenko, V. L’vov, A. Pomyalov, I. Procaccia, and G. Sahoo, Local and nonlocal energy spectra of superfuid ${ }^{3} \mathrm{He}$ turbulence, Phys. Rev. B 95, 184510 (2017).

[23] A. Kumar, M. K. Verma, and J. Sukhatme, Phenomenology of two-dimensional stably stratified turbulence under large-scale forcing, J. Turbulence 18, 219 (2017).

[24] A. Alexakis and L. Biferale, Cascades and transitions in turbulent flows, Phys. Rep. 767-769, 1 (2018).

[25] L. Biferale and I. Procaccia, Anisotropy in turbulent flows and in turbulent transport, Phys. Rep. 414, 43 (2005).

[26] L. Biferale, F. Bonaccorso, I. M. Mazzitelli, M. A. T. van Hinsberg, A. S. Lanotte, S. Musacchio, P. Perlekar, and F. Toschi, Coherent Structures and Extreme Events in Rotating Multiphase Turbulent Flows, Phys. Rev. X 6, 041036 (2016).

[27] B. Gallet, A. Campagne, P.-P. Cortet, and F. Moisy, Scaledependent cyclone-anticyclone asymmetry in a forced rotating turbulence experiment, Phys. Fluids 26, 035108 (2014).

[28] B. Gallet, Exact two-dimensionalization of rapidly rotating large-Reynolds-number flows, J. Fluid Mech. 783, 412 (2015).

[29] R. J. Donnelly and C. F. Barenghi, The observed properties of liquid helium at the saturated vapor pressure, J. Phys. Chem. Ref. Data 27, 1217 (1998).

[30] C. F. Barenghi, V. S. L'vov, and P.-E. Roche, Experimental, numerical, and analytical velocity spectra in turbulent quantum fluid, Proc. Natl. Acad. Sci. USA 111, 4683 (2014).

[31] L. Skrbek and K. R. Sreenivasan, Developed quantum turbulence and its decay, Phys. Fluids 24, 011301 (2012).
[32] E. Rusaouen, B. Chabaud, J. Salort, and P.-E. Roche, Intermittency of quantum turbulence with superfluid fractions from $0 \%$ to $96 \%$, Phys. Fluids 29, 105108 (2017).

[33] S. Babuin, V. S. L'vov, A. Pomyalov, L. Skrbek, and E. Varga, Coexistence and interplay of quantum and classical turbulence in superfluid He-4, Phys. Rev. B 94, 174504 (2016).

[34] L. Boue, V. S. L'vov, Y. Nagar, S. V. Nazarenko, A. Pomyalov, and I. Procaccia, Energy and vorticity spectra in turbulent superfluid He-4 from $T=0$ to $T_{\lambda}$, Phys. Rev. B 91, 144501 (2015).

[35] S. B. Pope, Turbulent Flows (Cambridge University Press, Cambridge, 2000).

[36] V. S. L'vov, S. V. Nazarenko, and G. E. Volovik, Energy spectra of developed superfluid turbulence, JETP Lett. 80, 535 (2004).

[37] J. Salort, B. Chabaud, E. Leveque, and P. E. Roche, Investigation of intermittency in superfluid turbulence, J. Phys.: Conf. Ser. 318, 042014 (2011).

[38] J. Salort, P. E. Roche, and Leveque, Mesoscale equipartition of kinetic energy in quantum turbulence, Europhys. Lett. 94, 24001 (2011).

[39] V. S. L'vov, S. V. Nazarenko, and L. Skrbek, Energy spectra of developed turbulence in helium superfluids, J. Low Temp. Phys. 145, 125 (2006).

[40] C. J. Gorter and J. H. Mellink, On the irreversible processes in liquid helium II, Physica 15, 285 (1949).

[41] L. Kondaurova, V. S. L'vov, A. Pomyalov, and I. Procaccia, Structure of a quantum vortex tangle in He-4 counterflow turbulence, Phys. Rev. B 89, 014502 (2014).

[42] D. N. McKinsey, C. R. Brome, J. S. Butterworth, S. N. Dzhosyuk, P. R. Huffman, C. E. H. Mattoni, J. M. Doyle, R. Golub, and K. Habicht, Radiative decay of the metastable $\operatorname{He}_{2}\left(a^{3} \Sigma_{u}^{+}\right)$molecule in liquid helium, Phys. Rev. A 59, 200 (1999).

[43] L. Biferale, M. Cencini, A. Lanotte, and D. Vergini, Inverse velocity statistics in two dimensions, Phys. Fluids 15, 1012 (2003).

[44] G. L. Eyink, Exact results on stationary turbulence in 2D: Consequences of vorticity conservation, Physica D 91, 97 (1991).

[45] V. S. L'vov and A. Pomyalov, Statistics of quantum turbulence in superfluid He, J. Low Temp. Phys. 187, 497 (2017).

[46] V. S. L'vov and I. Procaccia, The universal scaling exponents of anisotropy in turbulence and their measurement, Phys. Fluids 8 , 2565 (1996).

[47] I. Arad, V. S. L'vov, and I. Procaccia, Correlation functions in isotropic and anisotropic turbulence: The role of the symmetry group, Phys. Rev. E 59, 6753 (1999).

[48] I. Arad, V. S. L'vov, and I. Procaccia, Anomalous scaling in anisotropic turbulence, Physica A 288, 280 (2000).

[49] V. S. L'vov, I. Procaccia, and V. Tiberkevich, Scaling exponents in anisotropic hydrodynamic turbulence, Phys. Rev. E 67, 026312 (2003).

[50] Please notice that in Ref. [19] there is a typographical error referring to these data as $u_{\mathrm{s}, \mathrm{x}}^{2}$ and $u_{\mathrm{s}, \mathrm{y}}^{2}$. 